\title{
A LOCAL SOLUTION OF THE DIFFERENCE EQUATION $\Delta y(x)=F(x)$ AND OF RELATED EQUATIONS*
}

BY

I. M. SHEFFER

Introduction. We propose to consider certain aspects of the equation

$$
\Delta y(x) \equiv y(x+1)-y(x)=F(x),
$$

and of other equations to be mentioned. There have been three principal lines of study of (1): (a) that relating to special classes of functions $F(x)$; (b) that based on the character of $F(x)$ at infinity; and (c) that making a local study of the equation.

As an illustration of (a) is the theorem (established, by different methods, by Guichard, $\dagger$ Appell, Hurwitz, Carmichael) that if $F(x)$ is an entire function, then an entire function solution $y(x)$ exists. Again, if $F(x)$ is meromorphic, then a solution $y(x)$ exists which is also meromorphic (Hurwitz, loc. cit.).

Concerning (b): If the process of iteration be applied to (1), there arise two well known formal solutions $\ddagger$

$$
\begin{aligned}
& y(x) \sim \sum_{1}^{\infty} F(x-n), \\
& y(x) \sim-\sum_{0}^{\infty} F(x+n) .
\end{aligned}
$$

Unfortunately these series are too often divergent. But by the introduction of suitable exponential convergence factors, Nörlund has shown§ that the resulting series will converge for a large class of functions $F(x)$.

A local study of equation (1) was made by Guichard (loc. cit.) who set up a solution in the form of a definite integral (cf. Nörlund, loc. cit., p. 38). This integral, however, has the unfortunate feature of representing (in gen-

* Presented to the Society, September 6, 1934; received by the editors May 24, 1935, and, in revised form, October 25, 1935.

$\dagger$ References are to be found in Nörlund, Differenzenrechnung, 1924, especially pp. 38-39 and Bibliography; and in Carmichael, American Journal of Mathematics, vol. 35 (1913), pp. 163-182.

$\ddagger$ Cf., for example, Batchelder, Linear Difference Equations, Harvard University Press, 1927, p. 6.

$\S$ Loc. cit., pp. $40-43$ for a real variable, and pp. 68-71 for a complex variable. 
eral) an infinitely multiple-valued function. Some extensions of the local theory have been made by Carmichael (loc. cit.), using the Guichard integral.

Part I of the present paper is largely devoted to the local theory of equation (1). We obtain a solution in various forms, including a definite integral and a series of polynomials. In $\$ 1$ we give a general formal solution which includes (2) and (3), and state a sufficient condition under which convergence takes place in an infinite strip. The local study begins in $\$ 2$ where by use of the Pincherle integral* we show that if $F(x)$ is analytic in $|x|<r$ with $r>\frac{1}{2}$, then an analytic solution $y(x)$ exists in the neighborhood of $x=0$.

In $\S 3$ we consider the case where $F(x)$ is rational. Such functions have been treated before, but the particular form that we obtain for the solution (as a definite integral) is needed in the next section, $\$ 4$, where we introduce the polynomials $\left\{(x+1)^{n}-x^{n}\right\}$. Solutions of equation (1) are obtained as series in these polynomials, by various methods. Ultimately we are able to establish that every function $F(x)$ which is analytic about $x=-\frac{1}{2}$ has a convergent expansion (not however unique) in these polynomials; and corresponding to such a function $F(x)$, a solution is found.

Part II carries the methods of Part I over to the more general equation

$$
L[y(x)] \equiv a_{1} y\left(x+\omega_{1}\right)+\cdots+a_{k} y\left(x+\omega_{k}\right)=F(x) .
$$

In recent years this equation has been the subject of several investigations appearing in Acta Mathematica. $\dagger$ The point of view is that of Nörlund's principal solution, depending on the character of $F(x)$ at infinity, and using sum formulas. Our work consists in a local study of the equation, thus leading to results essentially different from those found in the papers mentioned.

\section{PART I}

1. A general formal solution. We seek a solution of equation

$$
y(x+1)-y(x)=F(x)
$$

* Our form of this integral is that used by Borel in his method of analytic continuation.

$\dagger$ S. Bochner, vol. 51 (1928), pp. 1-21: Hauptlösungen von Differenzengleichungen. finies.

R. Raclis, vol. 55 (1930), pp. 277-394: Solution principale de l'équation linéaire aux différences

M. Ghermanesco, vol. 62 (1934), pp. 239-287: Sur les équations aux différences finies.

For a real-variable treatment, consult S. Bochner, Vorlesungen über Fouriersche Integrale, Leipzig, 1932, chapters 5, 6.

Closer in spirit to our own work are the following important contributions:

Pincherle, Sur la résolution ..., Acta Mathematica, vol. 48 (1926), pp. 279-304. (This is a translation of an Italian memoir of 1888.)

Carmichael, Systems of linear difference equations . . , these Transactions, vol. 35 (1933), pp. 1-28, and Summation of functions . . , Annals of Mathematics, vol. 34 (1933), pp. 349-378. 
in the form*

$$
y(x)=\sum_{-\infty}^{\infty} L_{n}(x) F(x+n) .
$$

On substituting in (1.1) we find that

$$
L_{n-1}(x+1)-L_{n}(x)=\delta_{n 0}= \begin{cases}1, & n=0 \\ 0, & n \neq 0 .\end{cases}
$$

Let $L_{0}(x)$ be arbitrary. Then $L_{-n}(x)=1+L_{0}(x-n), \quad L_{n}(x)=L_{0}(x+n)$, $n=1,2, \cdots$, so that

TheOREM 1.1. A formal solution of (1.1) is given by

$$
y(x) \sim \sum_{0}^{\infty} L(x+n) F(x+n)+\sum_{1}^{\infty}[1+L(x-n)] F(x-n),
$$

where $L(x)$ is an arbitrary function.

Series (2), (3) of the Introduction are particular cases of (1.4), namely when $L(x)=0,-1$ respectively. The arbitrariness of $L(x)$ allows considerable freedom, and suggests that for many classes of functions $F(x)$, a choice of $L(x)$ may be made to yield convergence for (1.4), in which case (1.4) will define a solution of (1.1). In considering convergence, it is desirable that $L(x+n)$ and $1+L(x-n)$ approach zero rapidly as $n \rightarrow \infty$ (x remaining in some bounded region). We now examine a simple choice of $L(x)$.

Let $†$

$$
L(x)=-e^{-e^{x}},
$$

and consider an infinite strip $S$ parallel to the real axis:

$S$ :

$$
x=u+i v, \quad u \text { arbitrary, } \quad m \leqq v \leqq M,
$$

where $m, M$ are such that $\cos v \neq 0$ in $m \leqq v \leqq M$. Let $R$ be any bounded region

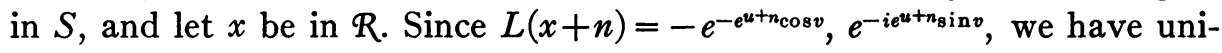
formly in $R$

$$
|L(x+n)| \leqq e^{-c e w+n} \quad(0<c \leqq \cos v, m \leqq v \leqq M) .
$$

On the other hand, $1+L(x-n)=\left[e^{e^{u-n+i v}}-1\right] \div e^{e^{u-n+i v}}$. The denominator approaches 1 uniformly ( $x$ in $R$ ) as $n \rightarrow \infty$, and may be ignored. It can then be

* Norlund (loc. cit., pp. 279-280) uses this device for a homogeneous equation of $n$th order. Consult also, in connection with the results of this section, E. Lindelöf, Le Calcul des Résidus, Paris, 1905; especially pp. 52-62.

$\dagger$ One can equally well replace $e$ by $a, a>1$. 
shown* that there exists a constant $B$ (depending only on $R$ ) such that (uniformly in $R$ )

$$
|1+L(x-n)| \leqq B e^{-n} .
$$

Relations (1.6) and (1.7) serve to establish

THEOREM 1.2. Let $F(x)$ be analytic except for isolated $\dagger$ singularities in a strip $S:$ u arbitrary, $m \leqq v \leqq M$, where c exists such that $0<c \leqq \cos v$ in $m \leqq v \leqq M$. Let $S^{\prime}$ denote a region obtained from $S$ by surrounding each singularity of $F(x)$ by a circle and removing the interior of the circle, and let there exist four positive numbers $r<e, \sigma>1, C, D$ (depending on $S^{\prime}$ ) such that for every $S^{\prime}$, and for all $x$ in $S^{\prime}$, the following relations hold:

$$
\begin{array}{ll}
|F(x)| \leqq C u^{-\sigma} \cdot e^{c e^{u}}, & \sigma>1, u>0 \\
|F(x)| \leqq D r^{|u|}, & r<e, u \leqq 0 .
\end{array}
$$

Then series (1.4), with $L(x)$ given by (1.5), converges for all $x$ in $S$ save (perhaps) for the singularities of $F(x)$ and all points conjugate $\ddagger$ (both right and left) to them. Moreover, (1.4) is a solution of (1.1) in $S$ (save at the points already excepted).

Theorem 1.2 is a sample of a type of theorem obtainable by a judicious choice of $L(x)$. Better theorems are surely possible, but it remains an open question if the ultimate theorem of this character can be obtained. It would state that whenever the rate of growth of $F(x)$ is known (in an $S$ strip) as $u \rightarrow \pm \infty$, then a corresponding $L(x)$ may be found such that (1.4) converges for all $x$ in $S$ save for certain points (and their conjugates) that are singularities of $F(x)$.

2. A local solution as a definite integral. In this section we shall establish, by use of the Pincherle (or Borel) integral and its inverse, that if $F(x)$ is analytic in $|x|<r$ where $r>\frac{1}{2}$, then a local solution exists.

Let $f(x)=\sum_{0}^{\infty} f_{n} x^{n} / n$ ! be an entire function of exponential type $\rho$ (exp.

* By the Law of the Mean applied to $e^{x}$ in $0 \leqq x \leqq e^{-n}$ we get $e^{e^{-n}}=1+e^{\xi_{n}-n}, 0<\xi_{n}<e^{-n}$. Hence

$$
H \equiv e^{u-n+i v}-1=\left(1+e^{\xi_{n}-n}\right) e^{u+i v}-1 .
$$

Since $e^{\xi_{n}-n}<1$, we may expand by the binomial theorem, getting

$$
\begin{aligned}
& \left.|H| \leqq A e^{-n}\left[\frac{C}{1 !}+A \frac{C(C+1)}{2 !} e^{-n}+\cdots\right] \quad C=\max \left|e^{u+i v}\right| \text { in } R, A=\max e^{-n}\right) \\
& |H| \leqq A C e^{-n}\left[1-A e^{-n}\right]^{-(C+1)} .
\end{aligned}
$$

From this (1.7) follows.

$\dagger$ The point at infinity may be a cluster point of singularities.

$\ddagger$ The points right (left) conjugate to $x$ are the points $x+1, x+2, \cdots(x-1, x-2, \cdots)$. 
type $\rho)$; i.e., lim sup $\left|f_{n}\right|^{1 / n}=\rho$, which we assume throughout to be finite. Then for every $x$, as is well known,*

$$
f(x)=\frac{1}{2 \pi i} \int_{C} \frac{F(1 / t)}{t} e^{t x} d t
$$

where $F(x)=\sum_{0}^{\infty} f_{n} x^{n}$, and $C$ is a contour about $t=0$ whose minimum distance from $t=0$ exceeds $\rho$.

Now the equation $\Delta y(x)=e^{t x}$ has an obvious solution $y(x)=e^{t x} \div\left(e^{t}-1\right)$, $t \neq \pm 2 k \pi i$. Applying this to (2.1) we get $\ddagger$

LEMMA 2.1. If $f(x)$ is of exp. type $\rho$, then a solution of

$$
\Delta y(x)=f(x)
$$

is given by

$$
y(x)=\frac{1}{2 \pi i} \int_{C} \frac{F(1 / t)}{t} \cdot \frac{e^{t x}}{e^{t}-1} d t
$$

where $C$ surrounds $t=0$, does not pass through any zero of $\left(e^{t}-1\right)$, and lies at a distance exceeding $\rho$ from $t=0$.

As simple deductions from Lemma 2.1 we have

Corollary 2.1. If the maximum distance from $C$ to $t=0$ is $\sigma$, then $y(x)$ of (2.3) is of exp. type not exceeding $\sigma$.

CoRollary 2.2. If $f(x)$ is of exp. type $\rho$, there is no solution $y(x)$ of exp. type less than $\rho$, but there is a solution of exp. type $\rho$. Such a solution is given by (2.3) where $C$ is the circle $|t|=\sigma$, with $2 k \pi \leqq \rho<\sigma<2(k+1) \pi$.

We have found a solution of the difference equation (2.2) for functions $f(x)$ that are of finite exponential type. This leaves out of consideration all entire functions of infinite exponential type, and all analytic functions that are not entire. We shall examine their case.

Let $F(x)=\sum f_{n} x^{n}$ be analytic about $x=0$, with radius of convergence $r$, and let $f(x)=\sum f_{n} x^{n} / n$ ! be the corresponding Pincherle entire function. (It is of exp. type $\rho=1 / r$.) The two functions are not only related by (2.1), but also by the Borel (or Pincherle) integral

* Pincherle, loc. cit., p. 285.

$\dagger F(x)$ is analytic at least in $|x|<1 / \rho$. We shall say that $f(x)$ is the Pincherle entire function associated with $F(x)$.

$\ddagger$ This result is to be found in Pincherle, loc. cit. See also Carmichael, Annals of Mathematics, loc. cit., pp. 361-367. 


$$
F(x)=\int_{0}^{\infty} e^{-t} f(t x) d t
$$

valid for every $x$ in the Borel polygon for $F(x)$.

Now consider the equation $\Delta y(x)=F(x)$. The trend of our argument is as follows: The function $f(t x)$ is (in $x$ ) of exp. type $|t| \rho$, so to it the preceding corollaries apply. We can then find a solution $y(x ; t)$ of $\Delta y(x ; t)=f(t x)$. As $t$ varies we shall need to revise the contour $C$ of (2.3). Passing over this (for the moment), we have, formally,

$$
F(x)=\int_{0}^{\infty} e^{-t} \Delta y(x ; t) d t=\Delta\left[\int_{0}^{\infty} e^{-t} y(x ; t) d t\right],
$$

so that a suggested solution of $\Delta y=F(x)$ is $y(x)=\int_{0}^{\infty} e^{-t} y(x ; t) d t$.

We can now validate the formal work. Divide the positive $t$-axis: $0 \leqq t<\infty$ into the intervals

$I_{n}$ :

$$
2(n-1) \pi \leqq t<2 n \pi \quad(n=1,2,3, \cdots) .
$$

For $t$ on $I_{n}$, set

$$
y_{n}(x ; t)=\frac{1}{2 \pi i} \int_{C_{n}} \frac{F(t / u)}{u} \cdot \frac{e^{u x}}{e^{u}-1} d u,
$$

where $C_{n}$ is the circle $|u|=2 n \pi r_{n}$, the numbers $r_{n}$ being chosen to fulfill the following two compatible conditions:

(i) $\sigma \geqq r_{n} \geqq \rho, n=1,2, \cdots$, where $\sigma, \rho$ are numbers satisfying $\sigma>\rho>1 / r$.

(ii) There is a number $\delta>0$ such that no pair of numbers, one from each of the two sets $\{2 m \pi\}, m=1,2, \cdots,\left\{2 n \pi r_{n}\right\}, n=1,2, \cdots$, are at a distance apart less than $\delta$.

For $t$ on $I_{n}$ and $u$ on $C_{n}$, we have $|t / u|<1 / r_{n}<r$, so that (by Lemma 2.1) (2.5) is a solution of $\Delta y=f(t x)$. Again, this inequality tells us that $|F(t / u)|$ is bounded, uniformly in $n$, for $t$ on $I_{n}$ and $u$ on $C_{n}$. This is also true* of the function $\left|e^{u}-1\right|^{-1}$. Consequently $y_{n}(x ; t)$ is of exp. type

$$
\leqq|u|=2 n \pi r_{n} \leqq 2 n \pi \sigma \leqq\left(\frac{n}{n-1}\right) t \sigma,
$$

$t$ on $I_{n}$; and $\left|y_{n}(x ; t)\right| \leqq N e^{\tau_{n}(x)}$, where $N$ is independent of $n, x, t$, and $\tau_{n}(x)=\max \{$ real part of $(u x)\}$ for $u$ on $C_{n}$.

We now define the function $y(x ; t)$ from $t=0$ to $t=\infty$ :

$$
y(x ; t)=y_{n}(x ; t), \quad t \text { on } I_{n} .
$$

* This follows by a straightforward analysis. 
TheOREM 2.1. Let $F(x)$ be analytic, $|x|<r$, and let $\tau$ be any number in $0<\tau<r$. Choose $\sigma, \rho$ so that $1 / \tau>\sigma>\rho>1 / r$, and determine the circles $C_{n}$ and the functions $y_{n}(x ; t)$ according to this $\sigma, \rho$. Then the function

$$
y(x)=\int_{0}^{\infty} e^{-t} y(x ; t) d t
$$

is analytic* in $|x|<\tau$.

For if $x$ is in $|x| \leqq \tau$, then

$$
\tau_{n}(x) \leqq 2 n \pi r_{n}|x| \leqq\left(\frac{n}{n-1}\right) t \sigma|x| \leqq\left(\frac{n}{n-1}\right) \gamma t,
$$

where $\gamma$ is chosen so that $\sigma \tau<\gamma<1$. Therefore (2.7) converges absolutely and uniformly in $|x| \leqq \tau$. We can write the integral as an infinite series:

$$
y(x)=\sum_{n=1}^{\infty} \int_{2(n-1) \pi}^{2 n \pi} e^{-t} y_{n}(x ; t) d t,
$$

which likewise converges absolutely and uniformly, $|x| \leqq \tau$. Moreover, each term is an analytic function in $|x| \leqq \tau$, thus making $y(x)$ analytic in $|x|<\tau$.

Now suppose $F(x)=\sum f_{n} x^{n}$ has a radius of convergence $r>\frac{1}{2}$. Choose $\tau$ as any number in $\frac{1}{2}<\tau<r$. There exist certain points $x$ in $|x|<\tau$ for which, also, $|x+1|<\tau$. Let $R$ be an open connected set (e.g., a small circle) of such points. For $x$ in $R$, we have from (2.8)

$$
\Delta y(x)=\sum_{1}^{\infty} \int_{2(n-1) \pi}^{2 n \pi} e^{-t} \Delta y_{n}(x ; t) d t=\sum_{1}^{\infty} \int_{2(n-1) \pi}^{2 n \pi} e^{-t} \cdot f(t x) d t=\int_{0}^{\infty} e^{-t} f(t x) d t,
$$

since $\Delta y_{n}=f(t x)$ on $I_{n}$. As the last integral is precisely $F(x)$, we see that $y(x)$ satisfies

$$
\Delta y(x)=F(x),
$$

at least for $x$ in $R$. Now $y(x)$ is analytic in $|x|<\tau$, so that $G(x)=y(x)+F(x)$ is also analytic there. Again, $G(x)=y(x+1)$ in $R$, whence, by analytic continuation, $y(x+1)$ is analytic in $|x|<\tau$; and this makes $y(x)$ a solution for all $x$ in $|x|<\tau$. We thus have

THEOREM 2.2. Let $F(x)$ be analytic in $|x|<r, r>\frac{1}{2}$. To every $\tau<r$ there corresponds a function $y(x)$, given by (2.8), which is analytic together with $y(x+1)$ in $|x|<\tau$, and which satisfies (2.9) in $|x|<\tau$.

The condition $\tau<r$ prevents us from asserting analyticity of $y(x)$ in the

$*|x|<\tau$ can be replaced by $|x| \leqq \tau$ by choosing $\tau^{\prime}$ slightly larger than $\tau$. 
larger region $|x|<r$. Inspection of the proof of Theorem 2.1 shows that the only purpose served by this condition is to guarantee that $|F(t / u)|$ is uniformly bounded, $t$ on $I_{n}$ and $u$ on $C_{n}$. There are cases when this is true even if $\tau=r$, in which case Theorem 2.2 will continue to hold:

COROLlaRy 2.3. If $F(x)$ is either analytic in $|x| \leqq r$ or is analytic and uniformly bounded in $|x|<r$, then the contours $C_{n}$ of (2.12) can be so chosen* that $y(x)$ as.given by (2.8) is analytic in $|x|<r$, and is a solution of (2.9) for all $x$ in $|x|<r$. (Here $r>\frac{1}{2}$.)

It is even possible, in certain cases, to remove the restriction that $|F(t / u)|$ be uniformly bounded:

CoRollary 2.4. Let $F(x)$ be analytic in $|x|<r$, where $r>\frac{1}{2}$. For each $\rho<r$, define $M(\rho)$ by

$$
M(\rho)=\max _{|x|=\rho}|F(x)| .
$$

If there exists an increasing sequence of positive numbers $\left\{\rho_{n}\right\}$ with $\rho_{n}<r$ and $\lim \rho_{n}=r$, such that to every $\epsilon>0$ there is a constant $A=A_{\epsilon}$ for which

$$
M\left(\rho_{n}\right) \leqq A e^{\dot{n} \epsilon},
$$

then $y(x)$ as given by $\dagger(2.8)$ is analytic in $|x|<r$, and is a solution of (2.9) throughout $|x|<r$.

The proof is quite straightforward, and may be omitted.

From the preceding theorem and corollaries follows

THeORem 2.3. Let $p(x)$ be a function for which a determination of $\log p(x)$ exists satisfying the hypotheses made on $F(x)$ in Theorem 2.2 or in Corollaries 2.3 or 2.4 . Then the equation

$$
y(x+1)-p(x) y(x)=0
$$

has a solution analytic in $|x|<\tau$ or $|x|<r$ according to the case.

In the theorems of this section it has been necessary to have $r>\frac{1}{2}$ in order that the difference operator be applicable to (2.8). This condition appears to

* We have only to replace condition (i) after (2.5) by (i) $r_{n}>1 / r$, with $r_{n} \rightarrow 1 / r$ as $n \rightarrow \infty$.

$\dagger$ With the same modification as in Corollary 2.3, choosing $r_{n}=1 / \rho_{n}$. There is, however, a point that needs clarification: Conditions (i), (ii) after (2.5) were compatible heretofore because the numbers $r_{n}$ were capable of variation (within limits). In the present case the sequence $r_{n}$ appears to be fixed, and conceivably condition (ii) is no longer true. However, we can always find a new sequence $\rho_{n}^{\prime}$ such that $\rho_{n}^{\prime} \leqq \rho_{n}, \rho_{n}^{\prime} \rightarrow r$, and such that (ii) is satisfied. Since $\rho_{n}^{\prime} \leqq \rho_{n},(2.11)$ also holds for $\rho_{n}^{\prime}$, and we may set $r_{n}=1 / \rho_{n}^{\prime}$. 
be essential for the method of this section, but it is not inherently necessary for the problem, as will be shown in $\$ 4$.

3. $F(x)$ rational. It is well known that if $F(x)$ is a rational function, the equation $\Delta y=F(x)$ has a solution that can be expressed in terms of the classi$\mathrm{cal}^{*} \Psi$-function and its derivatives. We shall then be brief in showing how a solution can be expressed as a definite integral. In $\$ 4$ we have need of the particular case where $F(x)=(x-\alpha)^{-1}$.

Let $F(x)=1 /(x-\alpha)=-(1 / \alpha) \cdot \sum_{0}^{\infty}(x / \alpha)^{n}, \alpha \neq 0$. The corresponding Pincherle entire function is $f(x)=(-1 / \alpha) e^{x / \alpha}$; and the work of the preceding section suggests that the function $\dagger$

$$
y(x)=y(x ; \alpha)=-\beta \int_{0}^{\infty} e^{-t} \frac{e^{t x \beta}-1}{e^{t \beta}-1} d t \quad\left(\beta=\frac{1}{\alpha}, \alpha \neq 0\right)
$$

is a solution of $\ddagger$

$$
\Delta y(x)=\frac{1}{x-\alpha} .
$$

Set $\beta=1 / \alpha=a+i b, a \neq 0$, and let $\rho=0$, for $a<0$, and $\rho=a$, for $a>0$. It is readily established that (3.1) converges absolutely for all $x=u+i v$ in the half-plane (i) $a u-b v-(1+\rho)<0$, and converges uniformly $\|$ in any closed region therein. Also, it diverges for every $x$ for which the left member of (i) is positive.

Let $l$ be the line through $\alpha$ perpendicular to the segment $0 \alpha$, and let $l^{(1)}$ be the line obtained by translating $l$ a distance one to the right. Then the half-plane of convergence (i) is determined by $l$ or $l^{(1)}$ according as $a<0$ or $a>0$. (In either case the origin lies in the half-plane.) On applying the difference operator to (3.1) we find that for every $x$ in the half-plane au-bv-1<0, (3.1) is a solution of (3.2).

Relations (3.1), (3.2) permit us to continue the function $y(x ; \alpha)$ analytically, giving

* $\Psi(x)=\Gamma^{\prime}(x) / \Gamma(x)$; cf. Batchelder, loc. cit., pp. 54-56. at $t=0$.

$\dagger$ We use $\left(e^{t x \beta}-1\right)$ in the numerator rather than $e^{t x \beta}$ in order to avoid convergence difficulties

$\ddagger$ For classical treatments of (3.2) (with $\alpha=0$, which makes no essential difference), see Nörlund, loc. cit., pp. 99-103; Carmichael, Annals of Mathematics, loc. cit., p. 359; and Milne-Thomson, The Calculus of Finite Differences, London, Macmillan, 1933, pp. 247-248. It is of interest to compare their form of solution with (3.1) and (3.3), (3.4).

$\S$ The condition $a \neq 0$ can be removed by a translation of the independent variable.

$\|$ There is uniform convergence in the half-plane $a u-b v-(1+\rho) \leqq-\delta$ for every $\delta>0$. 
THEOREM 3.1. For every positive integer $n$, the function $y(x ; \alpha)$ of $(3.1)$ has the representation

$$
\begin{aligned}
& y(x ; \alpha)=-\sum_{k=0}^{n-1} \frac{1}{(x+k-\alpha)}-\beta \int_{0}^{\infty} e^{-t} \cdot \frac{e^{t(x+n) \beta}-1}{e^{t \beta}-1} d t \quad(\alpha<0) ; \\
& y(x ; \alpha)=\sum_{k=1}^{n} \frac{1}{(x-k-\alpha)}-\beta \int_{0}^{\infty} e^{-t} \cdot \frac{e^{t(x-n) \beta}-1}{e^{t \beta}-1} d t \quad(\alpha>0) .
\end{aligned}
$$

The domain of convergence is a half-plane containing the origin and determined by the line ${ }^{(n)} l$ (by the line $\left.l^{(n+1)}\right)$, where ${ }^{(n)} l\left(l^{(n+1)}\right)$ is obtained from l by a translation $n$ units to the left $(n+1$ units to the right).

COROLLARY 3.1. If $a<0$ (if $a>0$ ) the only singularities of $y(x ; \alpha)$ are simple poles at the points $x=\alpha, \alpha-1, \alpha-2, \cdots$ (at the points $x=\alpha+1, \alpha+2, \cdots)$.

Having (3.3) or (3.4) it might be thought that by using Cauchy's formula we can now obtain a solution of $\Delta y(x)=F(x)$ for any analytic function. But this seemingly hopeful line proves illusory, ${ }^{*}$ and we are forced to modify this line of procedure.

If $F(x)$ is a rational function, a solution of the equation $\Delta y=F(x)$ can clearly be expressed in terms of the function $y(x ; \alpha)$ and its derivatives, where $\dagger \alpha$ takes on values corresponding to the poles of $F(x)$. Consequently the function $y(x ; \alpha)$ serves the same purpose as does the $\Psi$-function.

* (3.2) suggests that

$$
\Delta\left[-\frac{1}{2 \pi i} \int_{C} y(x ; \alpha) F(\alpha) d \alpha\right]=\frac{1}{2 \pi i} \int_{C} \frac{F(\alpha) d \alpha}{\alpha-x}=F(x),
$$

so that the bracket will be an analytic solution of $\Delta y=F(x)$. But it is not. For let $C$ be a circle lying (say) to the left of the imaginary axis. Let $F(x)$ be analytic in and on $C$. The integer $n$ can be chosen so large that the integral in (3.3) converges uniformly in $x$ and $\alpha$, for both $x$ and $\alpha$ in and on $C$. This integral is therefore analytic in $\alpha$ in and on $C$, and on multiplying by $F(\alpha)$ and integrating over $C$, the contribution is zero. Hence for $x$ interior to $C$,

$$
Y(x) \equiv-\frac{1}{2 \pi i} \int_{C} y(x ; \alpha) F(\alpha) d \alpha=\sum_{k=0}^{n-1} \frac{1}{2 \pi i} \int_{C} \frac{F(\alpha) d \alpha}{x+k-\alpha} .
$$

Now suppose for definiteness that $C$ is of radius less than $\frac{1}{2}$. Then of the points $\alpha=x, x+1, x+2, \cdots$, only $x$ is in $C$, so that $Y(x)=-F(x)$. Similarly,

$$
Y(x+1) \equiv-\frac{1}{2 \pi i} \int_{C} y(x+1 ; \alpha) F(\alpha) d \alpha=0,
$$

since none of the points $x+1, x+2, \cdots$ is in $C$. Hence while it is true that $Y(x+1)-Y(x)=F(x)$, yet $Y(x+1)$ is not the value of $Y(x)$ when $x$ is replaced by $x+1$. That is, we do not have an analytic solution.

$\dagger$ As stated before, the condition $a \neq 0$ (where $\beta=1 / \alpha=a+i b$ ) can be dispensed with. Further, if $F(x)$ is not zero at infinity, it is the sum of a polynomial and a rational function that is zero there. For the polynomial a polynomial solution always exists, while for the remaining rational function the treatment outlined above will serve. 
4. Solution in series of the polynomials $\left\{(x+1)^{n}-x^{n}\right\}$. In his proof that if $f(x)$ is an entire function, then $y(x)$, also entire, exists to satisfy $\Delta y=f(x)$, Hurwitz (loc. cit.) began with the Bernoulli polynomials, which satisfy the relations $\Delta B_{n}(x)=x^{n}\left(B_{n}\right.$ of degree $\left.n+1\right)$. If, then, $f(x)=\sum f_{n} x^{n}$, one is led to consider as a solution the series $\sum f_{n} B_{n}(x)$. Unfortunately, this series converges only for a limited class of entire functions. Hurwitz was therefore led to abandon a polynomial series, replacing it by a series of Bernoulli-Hurwitz functions. We wish to show in this section that there is a polynomial series in terms of which a solution for any analytic function can be expressed. The polynomials in question are $\left\{(x+1)^{n}-x^{n}\right\}, n=1,2, \cdots$.

THEOREM 4.1. If the series

$$
\sum_{1}^{\infty} c_{n}\left\{(x+1)^{n}-x^{n}\right\}
$$

converges* for $x=x_{0}$ where $\dagger R\left(x_{0}\right) \neq-\frac{1}{2}$, then it converges absolutely for every $x$ in the open region $\ddagger S$ common to the two circles $|x|<\rho_{0},|1+x|<\rho_{0}$, where $\rho_{0}=\max \left(\left|x_{0}\right|,\left|1+x_{0}\right|\right)$; and converges uniformly in every closed region in $S$, thus representing an analytic function in $S$.

The theorem follows readily on writing the series in the form

$$
\sum\left[c_{n}\left\{\left(x_{0}+1\right)^{n}-x_{0}{ }^{n}\right\}\right] \cdot\left[\left\{(x+1)^{n}-x^{n}\right\} \div\left\{\left(x_{0}+1\right)^{n}-x_{0}{ }^{n}\right\}\right] .
$$

ThEOREM 4.2. A necessary and sufficient condition that series (4.1) converge for at least one $x$ with $R(x) \neq-\frac{1}{2}$ is that lim sup $\left|c_{n}\right|^{1 / n}<2$.

(a) Suppose $\lim \sup \left|c_{n}\right|^{1 / n}<2$. Then $\delta$ exists in $0<\delta<2$ such that $\left|c_{n}\right|<(2-\delta)^{n}$ for all $n$ sufficiently large (say $\left.n \geqq N\right)$. Let $\epsilon>0$ be chosen to satisfy $2-\delta+\epsilon<2$, and choose $x_{0}=-(2-\delta+\epsilon)^{-1}$. Then $\left|x_{0}+1\right|<\left|x_{0}\right|$, so that $\left|c_{n}\left\{\left(x_{0}+1\right)^{n}-x_{0}{ }^{n}\right\}\right|<2\left|x_{0}\right|^{n}(2-\delta)^{n}, n \geqq N$. Now $\left|x_{0}\right|(2-\delta)<1$; hence (4.1) converges for $x=x_{0}$. (And $R\left(x_{0}\right) \neq-\frac{1}{2}$.)

* It suffices to have $\left|c_{n}\left\{\left(x_{0}+1\right)^{n}-x_{0}^{n}\right\}\right|$ bounded.

$\dagger$ That the condition $R\left(x_{0}\right) \neq-\frac{1}{2}$ is essential is seen as follows. Let $R\left(x_{0}\right)=-\frac{1}{2}$; then $x_{0}+1=\left|x_{0}\right| e^{i \omega_{0}}, x_{0}=\left|x_{0}\right| e^{i\left(\pi-\omega_{0}\right)}$, and

$$
\left(x_{0}+1\right)^{n}-x_{0}{ }^{n}= \begin{cases}\left|x_{0}\right|^{n}\left[2 i \sin n \omega_{0}\right], & n \text { even; } \\ \left|x_{0}\right|^{n}\left[2 \cos n \omega_{0}\right], & n \text { odd. }\end{cases}
$$

If, then, $\omega_{0}$ is commensurable with $\pi$, there will be infinitely many values of $n$ for which $\left\{\left(x_{0}+1\right)^{n}-x_{0}{ }^{n}\right\}=0$. For these values of $n$ we can choose $c_{n}$ as large as we please, with the result that there will be divergence of (4.1) for every $x$ not on $R(x)=-\frac{1}{2}$, while on $R(x)=-\frac{1}{2}$ there can be points of convergence.

$\ddagger$ The region of convergence (except possibly for points. on $R(x)=-\frac{1}{2}$ ) is then bounded by two circles of equal radii, with centers at $0,-1$. 
(b) Suppose (4.1) converges for $x=x_{0}, R\left(x_{0}\right) \neq-\frac{1}{2}$. By Theorem 4.1 we may assume that $\left|x_{0}\right|>\left|x_{0}+1\right|$. Then $\left|x_{0}\right|>\frac{1}{2}$, say $\left|x_{0}\right|=\delta+\frac{1}{2}, \delta>0$. If $\lim \sup \left|c_{n}\right|^{1 / n} \geqq 2$, then an $\epsilon>0$ exists for which both $\left(\frac{1}{2}+\delta\right)(2-\epsilon)>1$ and $\left|c_{n}\right|>(2-\epsilon)^{n}$ for infinitely many values of $n$, say $n=n_{1}, n_{2}, \cdots$. Consequently,

$$
\left|c_{n_{i}}\left\{\left(x_{0}+1\right)^{n_{i}}-x_{0}^{n_{i}}\right\}\right|>\frac{1}{2}(2-\epsilon)^{n_{i}}\left|x_{0}\right|^{n_{i}} \quad(i \text { sufficiently large }) .
$$

As the right hand member approaches infinity with $i$, series (4.1) fails to converge at $x=x_{0}$. This contradiction establishes the theorem.

The same type of argument serves to prove

Corollary 4.1. Let lim sup $\left|c_{n}\right|^{1 / n}=\rho<2$. Then series (4.1)

(i) converges absolutely for every $x$ in the region common to $|x|<1 / \rho$, $|1+x|<1 / \rho$

(ii) converges uniformly in every closed region therein;

(iii) diverges for every point $x$ exterior to this region and not lying on the line $R(x)=-\frac{1}{2}$.

For convenience we shall say that (4.1) converges only if it converges for at least one $x$ not on $R(x)=-\frac{1}{2}$; and if it converges in the region common to the circles $|x|<r,|1+x|<r$, we shall speak of such a region as a lens-region of convergence of radius $r$.

Expansions of type (4.1) can be related to solutions of equation (1). It is evident that the function $e^{t x}$ ( $t$ a parameter) has the expansion*

$$
e^{t x}=\sum_{1}^{\infty} \frac{t^{n}}{n !\left(e^{t}-1\right)}\left\{(x+1)^{n}-x^{n}\right\} \quad(t \neq \pm 2 k \pi i),
$$

convergent for all $x$, and uniformly convergent in $x$ and $t$ in any bounded $x$ region and any bounded $t$-region excluding arbitrarily small neighborhoods of the points $t= \pm 2 k \pi i$.

Series (4.2) can be integrated (in $t$ ) over any contour that avoids the points $\pm 2 k \pi i$. By use of (2.1) this tells us that if $f(x)$ is of exp.type $\rho$, then it has the expansion

$$
f(x)=\sum_{1}^{\infty}\left[\frac{1}{n !} \cdot \frac{1}{2 \pi i} \int_{C} \frac{F(1 / t)}{t} \cdot \frac{t^{n}}{e^{t}-1} d t\right]\left\{(x+1)^{n}-x^{n}\right\},
$$

* If (4.2) is multiplied by ( $\left.e^{t}-1\right)$, then no values of $t$ need be excluded. But now on setting $t= \pm 2 k \pi i$, we get a uniformly convergent expansion of the function zero. Hence an $\left\{(x+1)^{n}-x^{n}\right\}$ expansion is by no means unique; there are infinitely many expansions of zero: $0=\sum c_{n}\left\{(x+1)^{n}-x^{n}\right\}$, each one corresponding to a function $\sum c_{n} x^{n}$ that is periodic (with unity as a period) and analytic at least in $|x| \leqq \frac{1}{2}$. 
uniformly convergent in every bounded region. (Here $C$ is a contour about $t=0$, not passing through any point $t= \pm 2 k \pi i$, and with minimum distance from $t=0$ exceeding $\rho$.)

We now come to $F(x)$, assumed to be analytic in $|x|<r, r\rangle \frac{1}{2}$. Let $y(x)$ be the solution (2.8) of equation (2.9). As (2.8) converges uniformly in $|x| \leqq \tau$, $y(x)$ has a power series expansion $y(x)=\sum_{0}^{\infty} \lambda_{n} x^{n}$, whose coefficients $\lambda_{n}$ can be obtained by finding the corresponding coefficients in each function $\int_{2(n-1) \pi}^{2 n \pi} e^{-t} y_{n}(x ; t) d t$ and adding. Making use of $(2.5)$, we get

$$
\lambda_{n}=\frac{1}{n !} \sum_{k=1}^{\infty} \int_{2(k-1) \pi}^{2 k \pi} \frac{1}{2 \pi i} \int_{C_{k}} \frac{e^{-t} F(t / u) u^{n-1}}{e^{u}-1} d u d t .
$$

Since $\sum \lambda_{n} x^{n}$ converges in $|x| \leqq \tau$, so will $y(x+1)=\sum \lambda_{n}(x+1)^{n}$ converge in $|x+1| \leqq \tau$, and as we can choose $\tau>\frac{1}{2}$ (since $r>\frac{1}{2}$ ), the two power series for $y(x), y(x+1)$ (whose difference is $F(x)$ ) have a common domain of convergence. We accordingly have

TheOREM 4.3. Let $F(x)$ be analytic in $|x|<r, r>\frac{1}{2}$, and let $\tau$ be any number in $\frac{1}{2}<\tau<r$. Then $F(x)$ has the expansion

$$
F(x)=\sum_{1}^{\infty} \lambda_{n}\left\{(x+1)^{n}-x^{n}\right\},
$$

convergent in a lens-region of radius at least $\tau$. The coefficients $\lambda_{n}$ are given by (4.4), and $y(x)=\sum \lambda_{n} x^{n}$ is a solution of $\Delta y(x)=F(x)$ in the lens-region.

It is perhaps clear that Theorem 4.3 cannot be the best theorem relating to $\left\{(x+1)^{n}-x^{n}\right\}$-expansions, and for two reasons: $F(x)$ is here referred to the origin $(x=0)$ whereas Theorem 4.1 tells us that the central point in such expansions is the point $x=-\frac{1}{2}$; and an undue restriction is placed on the radius of convergence of $F(x)$. The result of $\$ 3$ will allow us to remove the non-essential conditions.

Consider again the function $y(x ; \alpha)$ of $(3.3), \alpha$ lying to the left of the imaginary axis. The only singularities of $y$ are at $x=\alpha, \alpha-1, \cdots$. Hence when $|\alpha|>\frac{1}{2}, y$ is analytic (about $x=0$ ) in a circle of radius greater than $\frac{1}{2}$; and we can write

$$
y(x ; \alpha)=\sum_{0}^{\infty} c_{n}^{\prime}(\alpha) x^{n} \quad \mid\left(\alpha \mid>\frac{1}{2}\right) .
$$

If $\alpha$ lies in a region $R$ such that $|\alpha| \geqq \rho>\frac{1}{2}$ uniformly in $R$, then (cf. (3.3)) series (4.6) converges uniformly in $x$ and $\alpha$ for $\alpha$ in $R$ and $x$ in $|x| \leqq \rho^{\prime}$ where $\rho^{\prime}$ is any number less than $\rho$. The functions $c_{n}^{\prime}(\alpha)$ are analytic in $R$.

The radius of convergence of (4.6) is no longer greater than $\frac{1}{2}$ if $|\alpha| \leqq \frac{1}{2}$. In this case let us proceed as follows: Define $\Omega$ by 


$$
\Omega(x ; \alpha)=\pi \cot \pi(x-\alpha) .
$$

The only singularities of this function are simple poles at $x=\alpha, \alpha \pm 1$, $\alpha \pm 2, \cdots$, and at $x=\alpha \pm n$ its principal part is $(x-\alpha \mp n)^{-1}$. Also, $\Delta \Omega(x ; \alpha)=0$. Hence the function

$$
Y(x ; \alpha)=y(x ; \alpha)+\Omega(x ; \alpha)
$$

satisfies (3.2), and its only singularities are simple poles at the points $x=\alpha+1, \alpha+2, \cdots$. Hence if $\delta>0$ is sufficiently small, then for all $\alpha$ satisfying both $|\alpha| \leqq \frac{1}{2}+\delta,|\alpha+1| \geqq \frac{1}{2}+\delta$, the nearest singularity of $Y$ to the origin is $x=\alpha+1$; so that

$$
Y(x ; \alpha)=\sum_{0}^{\infty} c_{n}^{\prime \prime}(\alpha) x^{n}
$$

$$
\left\{\begin{array}{l}
|\alpha| \leqq \frac{1}{2}+\delta \leqq|\alpha+1|, \\
\delta \text { sufficiently small, }
\end{array}\right.
$$

the series converging uniformly in $x$ and $\alpha$ for $x$ in $|x|<\delta^{\prime}\left(\delta^{\prime}<\frac{1}{2}+\delta\right)$ and $\alpha$ in the domain already described. And in this domain, $c_{n}^{\prime \prime}(\alpha)$ is analytic.

Let $\mathcal{L}$ be a lens-region of radius $r=\frac{1}{2}+\delta$, and let $A, B$ be the points in which the boundary $C$ (of $\mathcal{L}$ ) meets the line $R(x)=-\frac{1}{2}, A$ being the lower point. When $C$ is traversed in the positive sense, two arcs are described: $A B, B A$. Now $A B(B A)$ lies in the $\alpha$-domain for which $(4.9)((4.6))$ holds, so we have

Lemma 4.1. The function $(x-\alpha)^{-1}$ has the $\left\{(x+1)^{n}-x^{n}\right\}$-expansions

$$
\begin{aligned}
& \frac{1}{x-\alpha}=\sum_{n=0}^{\infty} c_{n}^{\prime \prime}(\alpha)\left\{(x+1)^{n}-x^{n}\right\}, \\
& \frac{1}{x-\alpha}=\sum_{n=0}^{\infty} c_{n}^{\prime}(\alpha)\left\{(x+1)^{n}-x^{n}\right\},
\end{aligned}
$$

uniformly convergent in $x$ and $\alpha$, for $x$ in every lens-region of radius less than $\frac{1}{2}+\delta$, and $\alpha$ on arcs $A B, B A$ respectively.

Now let $F(x)$ be a function analytic about $x=-\frac{1}{2}$, so that there is an open region $R$ of analyticity containing the point $-\frac{1}{2}$. A lens-region $\mathcal{L}$ can be chosen small enough so that it lies in $R$ and also so that for it Lemma 4.1 is valid. Let the boundary of $\mathcal{L}$ be $C=C^{\prime}+C^{\prime \prime}$, where $C^{\prime}, C^{\prime \prime}$ are the left and right half arcs. Then in $\mathcal{L}$,

$$
\begin{aligned}
F(x) & =\frac{1}{2 \pi i} \int_{C} \frac{F(\alpha)}{\alpha-x} d \alpha=\frac{1}{2 \pi i} \int_{C^{\prime}}+\frac{1}{2 \pi i} \int_{C^{\prime \prime}} \\
& =\sum_{1}^{\infty}\left\{-\frac{1}{2 \pi i} \int_{C^{\prime}} c_{n}^{\prime}(\alpha) F(\alpha) d \alpha-\frac{1}{2 \pi i} \int_{C^{\prime \prime}} c_{n}^{\prime \prime}(\alpha) F(\alpha) d \alpha\right\}\left\{(x+1)^{n}-x^{n}\right\},
\end{aligned}
$$

the convergence being uniform in every closed region in $\mathcal{L}$. We thus have 
THEOREM 4.4. If $F(x)$ is analytic about $x=-\frac{1}{2}$, it has an $\left\{(x+1)^{n}-x^{n}\right\}$ expansion valid in some lens-region $\mathcal{L}$ :

$$
\begin{aligned}
F(x) & =\sum_{1}^{\infty} f_{n}\left\{(x+1)^{n}-x^{n}\right\}, \\
f_{n} & =-\frac{1}{2 \pi i} \int_{C^{\prime}} c_{n}^{\prime}(\alpha) F(\alpha) d \alpha-\frac{1}{2 \pi i} \int_{C^{\prime \prime}} c_{n}^{\prime \prime}(\alpha) F(\alpha) d \alpha ;
\end{aligned}
$$

and the series $y(x)=\sum_{1}^{\infty} f_{n} x^{n}$ converges in a circle of radius exceeding $\frac{1}{2}$, and in this circle $y(x)$ is a solution of $\Delta y(x)=F(x)$.

If we combine Theorems 4.1 and 4.4 we can state

THEOREM 4.5. A necessary and sufficient condition that $F(x)$ have a (convergent) $\left\{(x+1)^{n}-x^{n}\right\}$-expansion $\dagger$ is that $F(x)$ be analytic about $x=-\frac{1}{2}$.

The special character of the point $-\frac{1}{2}$ is not inherent in the difference equation, as a translation shows. We thus get the following more general statement:

THEOREM 4.6. If $F(x)$ is analytic about the point $x=\alpha$, there is a function $y(x)$, analytic about $x=\alpha+\frac{1}{2}$ in a circle of radius exceeding $\frac{1}{2}$, which in this circle satisfies the difference equation $\Delta y(x)=F(x)$.

\section{PART II}

We shall now apply some of the methods of Part I to a more general equation. We shall give a local theory for the equation

$$
L[y(x)] \equiv a_{1} y\left(x+\omega_{1}\right)+a_{2} y\left(x+\omega_{2}\right)+\cdots+a_{k} y\left(x+\omega_{k}\right)=F(x),
$$

$k>1$, where no $a_{j}$ is zero and the $\omega$ 's are all distinct. The $a$ 's and $\omega$ 's are complex constants (some or all of which may be real).

5. Some geometric lemmas. We define $\rho_{j}(x), \rho(x)$ by

$$
\begin{aligned}
\rho_{j}(x) & =\left|x+\omega_{j}\right| \\
\rho(x) & =\max \left\{\rho_{1}(x), \cdots, \rho_{k}(x)\right\} .
\end{aligned} \quad(j=1, \cdots, k),
$$

The function $\rho(x)$ is continuous, and since $\rho(x) \rightarrow \infty$ as $|x| \rightarrow \infty$, it therefore has a minimum value $\rho^{*}$ :

$$
\rho^{*}=\min \rho(x) .
$$

$\dagger$ It would be of interest to determine the largest possible lens-region of convergence for a given function $F(x)$. 
Lемма 5.1. There is a unique point $\dagger x=x^{*}$ where $\rho(x)$ takes on its minimum value $\rho^{*}$.

An equivalent geometric statement is the following: Of all closed circles $C$ covering the set $P_{1}, \cdots, P_{k}$, where $P_{j}$ stands for the point $-\omega_{j}$, there is one and only one of smallest radius. We know there is at least one. Suppose there are two (say $C_{1}, C_{2}$ ) with radius $\rho^{*}$. Then $P_{1}, \cdots, P_{k}$, being covered both by $C_{1}$ and $C_{2}$, must lie in the closed zone common to $C_{1}, C_{2}$. But the circle $C$, on the common chord of $C_{1}, C_{2}$ as diameter, covers this zone and therefore covers $P_{1}, \cdots, P_{k}$; and its radius is less than $\rho^{*}$. This contradiction establishes the lemma.

Consider any two of the $k$ points, say $P_{j}=-\omega_{j}, P_{m}=-\omega_{m}$. Let $\perp_{j m}$ be the perpendicular bisector of segment $P_{j} P_{m}$. It defines two half-planes, and in that one containing $-\omega_{j}$ we have $\rho_{m}(x)>\rho_{j}(x)$, while on $\perp_{j m}$ we have $\rho_{m}(x)=\rho_{j}(x)$. Let $j$ be fixed, and draw the lines $\perp_{j 1}, \cdots, \perp_{j k}$. If for each line we choose that half-plane not containing $-\omega_{j}$, then the set of points common to all such half-planes constitutes the complete set $S_{i}$ at every point of which (and nowhere else) $\rho_{j}(x)$ is the only $\rho_{m}(x), m=1, \cdots, k$, having the maximum value $\rho(x)$. On the boundary of $S_{j}, \rho_{j}(x)$ shares this maximum with at least one other $\rho_{m}$. We denote the boundary by $B_{j}$. (One readily establishes that $B_{j}$ is a convex polygon with its end sides running off to infinity.)

We turn now to two lemmas that will be needed in $\$ 7$.

Lemma 5.2. $\ddagger$ Let $1^{\prime}, 2^{\prime}, 3^{\prime}$ be three points on a circle $C$, so situated that $2^{\prime}$ and $3^{\prime}$ are on opposite sides of the diameter through $1^{\prime}$, and such that chords $1^{\prime} 2^{\prime}$ and $1^{\prime} 3^{\prime}$ make angles less than $45^{\circ}$ with this diameter. Form a lattice work in the plane with $1^{\prime} 2^{\prime}$ and $1^{\prime} 3^{\prime}$ as adjacent sides of a lattice parallelogram. Then of all the lattice points in the plane, only $1^{\prime}, 2^{\prime}, 3^{\prime}$ are in or on $C$.

Lемма 5.3. In the figure of Lemma 5.2, let 1, 2, 3 be the points diametrically opposite $1^{\prime}, 2^{\prime}, 3^{\prime}$. Let $Q$ be any point in or on $C$ but distinct from any one of the six points $1, \cdots, 3^{\prime}$, and translate the lattice work of Lemma 5.2 so that $Q$ is a lattice point. If in this new lattice work there are lattice points other than $Q$ in or on $C$, then the only possibilities are the following:

$\dagger$ It may be asked where the minimum point $x^{*}$ lies. The following facts are readily ascertained. If there are exactly three points, $P_{1}, P_{2}, P_{3}$, then (i) $x^{*}$ is, the center of the circumscribing circle, and $\rho^{*}$ its radius, if $\Delta P_{1} P_{2} P_{3}$ is an acute-angled triangle; and (ii) $x^{*}$ is the mid-point of the longest side, and $\rho^{*}$ is half that side, in the contrary case. Now consider the general case of $k$ points: $P_{1}, \cdots, P_{k}$. Let $K$ be the smallest convex polygon covering this set. Each triad of vertices of $K$ determines an $x$ and a $\rho$, according to the rule just stated, and among these pairs $(x, \rho)$ is to be found the minimizing $\left(x^{*}, \rho^{*}\right)$.

$\ddagger$ The proof is quite elementary, and the lemma has been sent to the American Mathematical Monthly, to be proposed as a problem. 
(i) There are exactly two lattice points, $Q$ and $R$, where $Q R$ is parallel and equal to $1^{\prime} 2^{\prime}$ or parallel and equal to $1^{\prime} 3^{\prime}$.

(ii) There are two or more lattice points, all lying on a line l through $Q$, parallel to the shorter diagonals of the lattice work; and on l these points are consecutive lattice points.

Through $O$ (the center of $C$ ) draw a line parallel to $1^{\prime} 3^{\prime}$, and on this line choose two points $x_{3}, x_{4}$ so that $x_{3} 3^{\prime}=x_{4} 1^{\prime}=\rho$. Draw circles $C_{3}, C_{4}$ of radius $\rho$, centers $x_{3}, x_{4} . C_{3}$ and $C\left(C_{4}\right.$ and $\left.C\right)$ have in common a closed point set bounded by two arcs that intersect at $3^{\prime}$ and 1 (at $1^{\prime}$ and 3); call this region $R_{13^{\prime}}\left(R_{1^{\prime} 3}\right)$. $Q$ being in or on $C$, in order that a point $R$ exist, in or on $C$, such that $Q R$ is equal and parallel to $1^{\prime} 3^{\prime}$ (to $1^{\prime} 2^{\prime}$ ) it is necessary and sufficient that $Q$ be in $R_{13^{\prime}}$ or $R_{1^{\prime} 3}$ (in $R_{12^{\prime}}$ or $R_{1^{\prime} 2}$ ). $\dagger$

Let $Q$ be in $R_{13^{\prime}}$. We are to show that $R$ (in $R_{1^{\prime} 3}$ ) such that $Q R$ is equal and parallel to $1^{\prime} 3^{\prime}$ is the only other lattice point in or on $C$. There are two circles $C_{1}, C_{2}$ of radius $\rho$, having $Q R$ as a chord. Join $3^{\prime}$ to $Q$ by a straight line and imagine that $Q$ is now a variable point starting at $3^{\prime}$ and moving along to its original position. This effects a continuous translation on $C_{1}$; it starts in a position coincident with $C$, and ends in its original position. Now the farthest limiting position of $Q$ from $3^{\prime}$ is at 1 , and when $Q$ is at $1, C_{1}$ meet $C$ in 1 and 3 . Hence it follows by continuity that for any position of $Q$ in $R_{13^{\prime}}$ (but not at 1 or $3^{\prime}$ ), circle $C_{1}$ meets $C$ once interior to the arc $13^{\prime}$ and once interior to the arc $1^{\prime} 3$. A similar conclusion applies to $C_{2}$. Now $C_{3}\left(C_{4}\right)$ contains in and on it the whole arc $13^{\prime}\left(1^{\prime} 3\right)$. Hence all of $C$ (including boundary) is contained in $C_{1}+C_{2}+C_{3}+C_{4}$ (including boundary).

Let $S$ be any point common to $C, C_{3}$ (including boundary). Then $Q S<$ chord $13^{\prime}$, which is less than the minimum distance between any two lattice points; hence $S$ cannot be a lattice point. Similarly there are no lattice points in $R_{1^{\prime} 3}$ other than $R$. As for $C_{1}$ : If through $R$ we draw a line parallel to $1^{\prime} 2^{\prime}$, it cuts $C_{1}$ in a point $S_{1}$ which is a lattice point. By Lemma 5.2, $S_{1}, Q, R$ are the only lattice points in or on $C_{1}$. (Similarly, there is a point $S_{2}$ on $C_{2}$ such that $S_{2}, Q, R$ are the only lattice points in or on $C_{2}$.) But $S_{1}$ and $S_{2}$ are exterior to $C$. For consider $S_{1}$, say. If it is in or on $C$, then since $R S_{1}$ is equal and parallel to $1^{\prime} 2^{\prime}$, therefore $R$ must be either in $R_{12^{\prime}}$ or $R_{1^{\prime 2}}$; a contradiction.

This establishes the first part of the lemma. The second part likewise follows by a geometric continuity argument.

$\dagger R_{12^{\prime}}, R_{1^{\prime} 2}$ are the closed regions common to $C$ and $C_{6}$ and to $C$ and $C_{6}$ respectively, where $C_{5}, C_{6}$ are circles of radius $\rho$ and centers $x_{5}, x_{6}$, and where $x_{5}, x_{6}$ lie on the line through $O$ parallel to $1^{\prime} 2^{\prime}$ and such that $C_{5}\left(C_{6}\right)$ passes through 1 and $2^{\prime}\left(1^{\prime}\right.$ and 2$)$. 
6. The $A_{n}(x)$-series and a solution of $L[y(x)]=1 /(x-\alpha)$. We introduce the polynomials $\dagger$

$$
A_{n}(x)=L\left[x^{n}\right]=a_{1}\left(x+\omega_{1}\right)^{n}+\cdots+a_{k}\left(x+\omega_{k}\right)^{n} \quad(n=0,1, \cdots) .
$$

The determination of an asymptotic expression of $A_{n}(x)$ is possible if no two of the quantities $\rho_{j}(x)=\left|x+\omega_{j}\right|, j=1, \cdots, k$, give the maximum value $\rho(x)$. Now we saw that the maximum $\rho(x)$ is shared when and only when $x$ is on a boundary $B_{j}$. We shall therefore define the set of boundary points $\left(B_{1}+\cdots+B_{k}\right)$ as the critical set. $f$

THEOREM 6.1. If the series $\sum_{0}^{\infty} c_{n} A_{n}(x)$ converges at $x=x_{0}$ (not in the critical set), it converges absolutely in the circular polygon $\Lambda_{0}$ defined by

$$
\Lambda_{0}: \quad\left|x+\omega_{j}\right|<\rho\left(x_{0}\right) \quad(j=1,2, \cdots, k) ;
$$

and converges uniformly in every closed region therein, thus representing an analytic function in $\Lambda_{0}$. The region $\Lambda_{0}$ contains the point $x=x^{*}$.

The theorem follows from the fact that a constant $m$ exists such that $m\left[\rho\left(x_{0}\right)\right]^{n} \leqq\left|A_{n}\left(x_{0}\right)\right|$, and that for $x$ in any closed region in $\Lambda_{0}$, there exist numbers $C, \rho^{\prime}$ such that $\left|A_{n}(x)\right| \leqq C \rho^{\prime n}, \rho^{\prime}<\rho\left(x_{0}\right)$.

THEOREM 6.2. A necessary and sufficient condition that the series $\sum_{0}^{\infty} c_{n} A_{n}(x)$ converge for at least one point not in the critical set is that lim $\sup \left|c_{n}\right|^{1 / n}<1 / \rho^{*}$.

(a) Suppose the series converges for $x=x_{0}$ not in the critical set. Then there is just one value $j$ for which $\rho_{j}\left(x_{0}\right)=\rho\left(x_{0}\right)$. Call this common value $\rho^{\prime}$. If it is false that $\lim \sup \left|c_{n}\right|^{1 / n}<1 / \rho^{*}$, then there exists an $\epsilon>0$ such that $\left|c_{n}\right|>\left(1 / \rho^{*}-\epsilon\right)^{n}$ for infinitely many values of $n$, say $n_{1}, n_{2} \cdots$. Now $M$ exists so that $\left|A_{n}\left(x_{0}\right)\right|>M \rho^{\prime n}, n>N$ (suitably chosen). Hence

$$
\left|c_{n_{i}} A_{n_{i}}\left(x_{0}\right)\right|>M\left(\frac{1}{\rho^{*}}-\epsilon\right)^{n_{i}} \cdot \rho^{\prime n_{i}}
$$

But $\rho^{\prime}>\rho^{*}$, so that for $\epsilon$ sufficiently small the bracket is greater than unity. The series therefore diverges for $x=x_{0}$; a contradiction.

(b) Suppose $\lim \sup \left|c_{n}\right|^{1 / n}=\sigma<1 / \rho^{*}$. Then $\delta$ exists on $0<\delta<1 / \rho^{*}$ such that $\left|c_{n}\right|<\left(1 / \rho^{*}-\delta\right)^{n}$ for all $n \geqq N$. Choose $x_{0}=x^{*}+\gamma$ where $|\gamma|<\epsilon$, and (as

$\dagger$ This set is a natural generalization of the polynomial set $\left\{(x+1)^{n}-x^{n}\right\}$. It is mentioned by Ghermanesco (loc. cit., p. 249), but he scarcely makes use of the set. Pincherle (loc. cit., pp. 282-284) points out the relation of Appell polynomials (of which $\left\{A_{n}(x)\right\}$ is an example) to certain linear functional equations (including the one we are studying).

$\ddagger$ The convergence problem on the critical set involves difficulties of a kind already encountered at the beginning of $\S 4$, the critical set there being the line $R(x)=-\frac{1}{2}$. 
is possible) such that $x_{0}$ is not in the critical set. The point $x^{*}$ is in the critical set; suppose it is on $B_{j}$. Then

$$
\sum\left|c_{n} A_{n}\left(x_{0}\right)\right| \leqq M \sum\left(\frac{1}{\rho^{*}}-\delta\right)^{n} \cdot\left|x_{0}+\omega_{j}\right|^{n} \leqq M \sum\left(\frac{1}{\rho^{*}}-\delta\right)^{n} \cdot\left(\rho^{*}+\epsilon\right)^{n} .
$$

On choosing $\epsilon$ sufficiently small, this last series converges; hence so does the original at $x=x_{0}$.

Corollary 6.1. If $\lim \sup \left|c_{n}\right|^{1 / n}=\sigma<1 / \rho^{*}$, then $\sum c_{n} A_{n}(x)$ converges absolutely for every $x$ in the (open) circular polygon of radius $1 / \sigma$; converges uniformly in every closed region therein; and diverges for every $x$ exterior to this polygon (save possibily for points in the critical set).

We are concerned with the equation

$$
L[y(\alpha)]=\alpha_{1} y\left(x+\omega_{1}\right)+\cdots+\alpha_{k} y\left(x+\omega_{k}\right)=F(x) .
$$

From (6.3) we get

$$
L\left[e^{t x}\right]=L(t) e^{t x},
$$

where

$$
L(t)=\alpha_{1} e^{\omega_{1} t}+\cdots+\alpha_{k} e^{\omega_{k} t} .
$$

It follows that for every $t$ for which $L(t) \neq 0$, equation $L[y(x)]=e^{t x}$ has $y(x)=e^{t x} / L(t)$ as a solution; and the expansion $e^{t x}=\sum_{0}^{\infty}\left\{\left[t^{n} A_{n}(x)\right] \div[n ! L(t)]\right\}$ is valid for all $x$.

From this we get (cf. series (4.3) of $\S 4$ )

THEOREM 6.3. $†$ If $f(x)$ is of exp. type $\lambda$, then it has the expansion

$$
f(x)=\sum_{0}^{\infty}\left[\frac{1}{n !} \cdot \frac{1}{2 \pi i} \int_{C} \frac{F(1 / t)}{t} \cdot \frac{t^{n}}{L(t)} d t\right] A_{n}(x),
$$

uniformly convergent in every bounded region. $\ddagger$

Zeros of $L(t)$ give rise to expansions of zero, corresponding to exponential solutions of the homogeneous $L$-equation. Thus, if $L\left(t_{1}\right)=0$, then $e^{t_{1} x}$ satisfies $L[y(x)]=0$, and zero has the convergent expansion $0=\sum_{0}^{\infty}\left(t_{1}^{n} / n !\right) A_{n}(x)$.

To investigate the possibility of expanding an "arbitrary" analytic function in an $A_{n}$-series, we are led to introduce the equation

† See Carmichael, these Transactions, loc. cit., pp. 1-7.

$\ddagger C$ is a contour about $t=0$, not passing through any zero of $L(t)$, and with minimum distance from $t=0$ exceeding $\lambda$. 


$$
L[y(x ; \alpha)]=\frac{1}{x-\alpha} ;
$$

and the work of $\$ 3$ suggests the solution $\dagger$

$$
y_{1}(x ; \alpha)=-\beta \int_{0}^{\infty} e^{-t} \cdot \frac{e^{\beta t x}}{L(\beta t)} d t, \quad \beta=\frac{1}{\alpha} .
$$

It is indeed a formal solution, and, if convergent, will be a true solution. There will be a convergence difficulty at $t=0$ if $L(0)=0$. In this case, suppose $t=0$ is a $p$-fold zero of $L(t)$. Then if we replace (6.8) by

$$
y_{2}(x ; \alpha)=-\beta \int_{0}^{\infty} e^{-t} \cdot \frac{e^{\beta t x}-\sum_{r=0}^{p-1}(t \beta x)^{r} / r !}{L(\beta t)} d t,
$$

the integrand is convergent at the origin-end of the interval. This may however restrict the range of $x$ for which convergence takes place at the other end. To avoid this we write

$$
\begin{aligned}
y(x ; \alpha)= & -\beta \int_{0}^{T} e^{-t} \cdot \frac{e^{\beta t x}-\sum_{r=0}^{p-1}(t \beta x)^{r} / r !}{L(\beta t)} d t \\
& -\beta \int_{T}^{\infty} e^{-t} \cdot \frac{e^{\beta t x}}{L(\beta t)} d t
\end{aligned}
$$

where $T$ is any fixed positive number (which, however, we choose as zero if $L(0) \neq 0)$.

Define $\sigma_{j}(\alpha), \sigma(\alpha)$ by

$$
\begin{aligned}
\sigma_{j}(\alpha) & =R\left(\beta \omega_{j}\right)=R\left(\omega_{j} / \alpha\right) \quad(j=1, \cdots, k), \\
\sigma(\alpha) & =\max \left\{\sigma_{1}(\alpha), \cdots, \sigma_{k}(\alpha)\right\} .
\end{aligned}
$$

If for a given $\alpha$, the maximum, $\sigma(\alpha)$, is attained for only one value of $j$, then $L(\beta t)$ is of order $e^{t \sigma(\alpha)}$. But if two or more indices $j$ give the maximum, the order of $L(\beta t)$ may not be clearly determined. If possible, then, we should avoid such values of $\alpha$. Now if $\alpha$ is of this character, so also is $c \alpha$, where $c$ is any positive number. We thus get a ray of values $\alpha$ (issuing from the origin)

† Pincherle (loc. cit., pp. 289-295) uses an analogous integral to discuss the equation $L[y(x)]=F(x)$ where $F(x)$ is a function analytic at infinity. See also his pages 295-297 for an interesting integral and series in the special case that $L(t)$ has the form

$$
L(t)=\left(1-h_{1} e^{\alpha_{1} t}\right)\left(1-h_{2} e^{\alpha_{2} t}\right) \cdots\left(1-h_{m} e^{\alpha_{m} t}\right) .
$$


of this type. The set of such rays (of which there are only a finite number) we shall term the set of primary critical rays.

It is readily established $\dagger$ that if $P_{1}, \cdots, P_{k}$ are the points of Lemma 5.1, then the primary critical rays are to be found among those half-lines (i.e., rays) drawn from the origin perpendicular to the segments $P_{j} P_{r}, j \neq r=1, \cdots, k$. The primary rays divide the plane into sectors, and throughout each sector there is one and only one $j$ for which $\sigma_{j}(\alpha)=\sigma(\alpha)$. We shall assume that $\alpha$ is not on a primary ray. $\neq$ Then, as has been pointed out, $L(\beta t)$ is of order $e^{t \sigma(\alpha)}$, so that we have

LEMMa 6.1. For $\alpha$ not on a primary or secondary critical ray, the integral (6.10) converges for all $x$ in the half-plane $R(-1+\beta x-\sigma(\alpha))<0$. This halfplane is bounded by the line perpendicular to the line joining $\alpha$ to the origin, meeting this latter line in the point $[1+\sigma(\alpha)] \alpha$; and is that half-plane indicated by an arrow from $\alpha$ pointing toward the origin.

Let $l$ denote the boundary line of the half-plane of convergence. If we replace $x$ by $x+\omega_{j}$ in (6.10), convergence takes place in a half-plane whose boundary line $l_{i}$ is obtained from $l$ by a translation $\S$ of $-\omega_{j}$. In this way we get $k$ half-planes bounded by parallel lines $l_{1}, \cdots, l_{k}$. In that half-plane common to all the $k$ half-planes, the operator $L$ can be applied to $y(x ; \alpha)$, and the result is $1 /(x-\alpha)$. That is,

Lemma 6.2. The function $y(x ; \alpha)$ of (6.10) satisfies (6.7) in the half-plane $R(-1+\beta x)<0$, which is determined by that one of $l_{1}, \cdots, l_{k}$ that passes through $\alpha$.

$y(x ; \alpha)$ will remain a solution of $(6.7)$ in every region into which it can be continued. We now examine such continuation. The point $\alpha$ not being on a critical ray, it must lie in a sector bounded by primary critical rays. In this sector there is one and only one index, say $j=M$, for which $\sigma_{j}(\alpha)=\sigma(\alpha)$; and of $l_{1}, \cdots, l_{k}$, it is $l_{M}$ which passes through the point $\alpha$. The remaining lines lie on that side of $l_{M}$ that is away from the origin. Denote by $H_{j}$ the half-plane (containing the origin) bounded by $l_{j}$. The functions $y\left(x+\omega_{j}\right), j=1, \cdots, k$, are analytic in $H_{j}$, and therefore all these functions are analytic in $H_{M}$.

† Cf. Pincherle, loc. cit., pp. 289-291.

$\ddagger$ Also, we must avoid those values $\alpha$ for which $L(\beta t)$ vanishes somewhere on $0<t<\infty$. If $t_{1}, t_{2}, \cdots$ are the zeros of $L(t)$, then no one of the numbers $\alpha t_{n}, n=1,2, \cdots$, is to be real and positive. If we draw the ray issuing from the origin and passing through $t_{n}$, and then reflect in the real axis, we get a ray on which $\alpha$ must not lie. Such a ray we shall term a secondary critical ray.

$\S$ The equation of the half-plane is $R\left(-1+\beta x+\sigma_{j}(\alpha)-\sigma(\alpha)\right)<0$. The smallest value that $\sigma(\alpha)-\sigma_{j}(\alpha)$ has, $j=1,2, \cdots, k$, is 0 , so that the "smallest" half-plane is given by $R(-1+\beta x)<0$. This half-plane is determined by the line through $\alpha$, and it contains the origin. 
Let us solve (6.7) for $y\left(x+\omega_{M}\right)$ :

$$
y\left(x+\omega_{M}\right)=\frac{1}{\alpha_{M}}\left\{\frac{1}{x-\alpha}-\sum_{j=1}^{k} a_{j} y\left(x+\omega_{j}\right)\right\},
$$

where the prime indicates that $j=M$ is excepted. Now of the lines $l_{i}(j \neq M)$, one $\dagger$ of them, say $l_{1}$, is nearest to $l_{M}$. The right hand side of (6.13) is therefore analytic in $H_{1}$, save for a simple pole at $x=\alpha$. That is, except for $x=\alpha$, the region of analyticity of $y\left(x+\omega_{M}\right)$ has been extended from $H_{M}$ to $H_{1}$. This automatically extends the range of $y\left(x+\omega_{j}\right)$, except for a pole, from $H_{j}$ to $H_{j}{ }^{(1)}$ (as we shall term it), where $H_{j}^{(1)}$ is the half-plane defined by the line $l_{j}^{(1)}$, obtained from $l_{j}$ by the translation $\ddagger \omega_{M}-\omega_{1}$. At $x=\alpha+\omega_{M}-\omega_{j}$ (which is on $\left.l_{j}\right), y\left(x+\omega_{j}\right)$ has a simple pole of residue $1 / a_{M}$. Elsewhere in $H_{j}^{(1)}$ it is analytic. The half-planes $H_{j}^{(1)}$ have $H_{M}{ }^{(1)}=H_{1}$ in common. Also, for $j \neq M$, they have $H_{1}{ }^{(1)}$ in common, and $y\left(x+\omega_{j}\right), j \neq M$, is analytic in $H_{1}^{(1)}$ except that for some $\S$ values of $j$ there may be simple poles with residue $1 / a_{M}$. This permits us to use (6.13) again.

The result is to extend the region of analyticity of $y\left(x+\omega_{M}\right)$ (save for certain poles) by another translation of amount $\omega_{M}-\omega_{1}$; and the same thing applies to $y\left(x+\omega_{j}\right)$. Let the boundaries of the new half-planes $\| H_{j}^{(2)}$ be $l_{j}^{(2)}$, obtained by the translation $\omega_{M}-\omega_{1}$ from $l_{j}^{(1)}$. As for the poles of $y\left(x+\omega_{M}\right)$ in $H_{M}(2)$ : we have already noted the simple pole at $x=\alpha$. There is clearly a simple pole at $x=\alpha+\omega_{M}-\omega_{1}$, with residue $-a_{1} / a_{M}{ }^{2}$. The only other poles in $H_{M}^{(2)}$ come from those values of $j$ for which the pole $\alpha+\omega_{M}-\omega_{j}$ of $y\left(x+\omega_{j}\right)$ lies in $H_{M}^{(2)}$; in which case $\alpha+\omega_{M}-\omega_{j}$ is a simple pole with residue $-a_{j} / a_{M^{2}}$. For each pole of $y\left(x+\omega_{M}\right)$ in $H_{M}^{(2)}$ we get a corresponding (simple) pole of $y\left(x+\omega_{j}\right)$ in $H_{j}^{(2)}$, with the same residue.

This process can be carried out indefinitely, each time extending the region by the translation $\omega_{M}-\omega_{1}$. In general we have half-planes $H_{j}^{(n)}$, bounded by $l_{j}^{(n)}$, where

$$
H_{M^{(n)}}=H_{1}^{(n-1)}, l_{M}^{(n)}=l_{1}^{(n-1)} .
$$

Each time that a pole of $y\left(x+\omega_{j}\right), j \neq M$, enters for the first time a region

$\dagger$ There may be several lines coincident with $l_{1}$; but no line $l_{j}(j \neq M)$ coincides with $l_{M}$.

$\ddagger$ The point $x=\alpha$ we know to be on $l_{M}$. It is easily computed that $x=\alpha+\omega_{M}-\omega_{j}$ is on $l_{j}$. The lines $l_{1}, \cdots, l_{k}$ can then be characterized as follows: They are the lines through $\alpha+\omega_{M}-\omega_{j}$, $j=1, \cdots, k$, perpendicular to the line joining $\alpha$ to the origin. When we move from $l_{M}$ to $l_{M}{ }^{(1)}=l_{1}$, which is a translation by the vector $\omega_{M}-\omega_{1}$, each of the lines $l_{j}$ undergoes a translation of the same amount.

$\S$ These values of $j$ will correspond to those points $\alpha+\omega_{M}-\omega_{j}(j \neq M)$ that lie in $H_{1}^{(1)}$.

$\| H_{M^{(2)}}=H_{1}^{(1)}, l_{M}^{(2)}=l_{1}^{(1)}$. 
$H_{M}^{(n)}$, it provides a pole for $y\left(x+\omega_{M}\right)$ at the same point, whose residue is promptly multiplied by $-a_{i} / a_{M}$. In the light of this discussion it becomes clear that we have

THEOREM 6.4. For each $\alpha$ in a sector $\dagger$ bounded by primary critical rays (and $\alpha$ not on a secondary ray), the function $y(x ; \alpha)$ is a meromorphic function. Its only poles are simple, and they are at the points $\ddagger$

$$
\begin{aligned}
& x=\alpha+\omega_{M} ; \quad x=\alpha+2 \omega_{M}-\omega_{j_{1}} \quad\left(j_{1}=1, \cdots, k ; j_{1} \neq M\right) ; \cdots ; \\
& x=\alpha+(n+1) \omega_{M}-\left(\omega_{j_{1}}+\cdots+\omega_{j_{n}}\right)
\end{aligned}
$$

$$
\left(j_{1}, \cdots, j_{n}=1, \cdots, k ; j_{1} \neq M, j_{2} \neq M, \cdots, j_{n} \neq M\right) ;
$$

and the corresponding residues are

$$
\frac{1}{a_{M}} ; \frac{-a_{j_{1}}}{a_{M}} ; \frac{a_{j_{1}} a_{j_{2}}}{a_{M}} ; \cdots ; \frac{(-1)^{n} a_{j_{1}} \cdots a_{j_{n}}}{a_{M}{ }^{n+1}} .
$$

We can now treat equation (6.3) in the case that $F(x)$ is rational.

Lemma 6.3. If $F(x)$ is a polynomial, then (6.3) has a polynomial solution.

To show this, we observe that if $L(t)$ has a $p$-fold zero at $t=0$ ( $p=0$ is possible) then $A_{n}(x)$ is of degree exactly $n-p, n \geqq p .\left(A_{n}(x) \equiv 0, n<p\right.$.) Let $F(x)$ be of degree $r$. Then $F(x)$ has an expansion $F(x)=\sum_{i=0}^{r} f_{i} A_{p+i}(x)$, and $y(x)=\sum_{i=0}^{r} f_{i} x^{p+i}$ is a solution. $\S$

Now let $F(x)$ be a rational function. By virtue of Lemma 6.3 we can suppose that $F(x)$ is analytic and zero at $x=\infty$, so that it has the form

$$
R(x)=\sum_{j=1}^{s} \sum_{r=1}^{m_{j}} \frac{f_{j r}}{\left(x-\alpha_{j}\right)^{r}}
$$

Now

$$
L\left[\frac{(-1)^{r-1}}{(r-1) !} \frac{d^{r-1}}{d x^{r-1}} y(x ; \alpha)\right]=\frac{1}{(x-\alpha)^{r}}
$$

which gives us

$\dagger$ The same index $M$ is retained for all values $\alpha$ in one and the same sector. We may call it a $\Sigma_{M}$-sector.

$¥$ The singularity of $y(x ; \alpha)$ nearest the origin is at $x=\alpha+\omega_{M}$.

$\S$ If $p \neq 0$, the most general polynomial solution is obtained by adding to $y(x)$ the polynomial $c_{0}+c_{1} x+\cdots+c_{p-1} x^{p-1}$, where the $c$ 's are arbitrary. If $p=0$, there is a unique polynomial solution. 
THEOREM 6.5. If $F(x)$ is a rational function, the equation (6.3) has a meromorphic solution. If all its poles lie in the same sector bounded by primary rays, and none of its poles is on a secondary ray, then on writing $F(x)=P(x)+R(x)$, where $P(x)$ is a polynomial and $R(\infty)=0$ (so that $R(x)$ is given by (6.14)), this solution can be written

$$
y(x)=y_{0}(x)+\sum_{j=1}^{s} \sum_{r=1}^{m_{j}} \frac{(-1)^{r-1} f_{j r}}{(r-1) !} \frac{d^{r-1} y\left(x ; \alpha_{j}\right)}{d x^{r-1}} .
$$

Here $y_{0}(x)$ is any polynomial solution of $L\left[y_{0}\right]=P(x)$ assured us by Lemma 6.3. If the poles are not so located, then a constant $\gamma$ can be found so that the solution can be written

$$
y(x)=y_{0}(x)+\sum_{j=1}^{s} \sum_{r=1}^{m_{j}} \frac{(-1)^{r-1} f_{j r}}{(r-1) !} \frac{d^{r-1} y\left(x+\gamma ; \alpha_{i}+\gamma\right)}{d x^{r-1}} .
$$

The theorem is evident if the poles $\alpha_{j}$ are in one sector and are off secondary rays. In the contrary case a number $\gamma$ can be found such that the points $\alpha_{j}+\gamma$ satisfy the previous condition. Set $G(x)=R(x-\gamma)$. Its poles are at $\alpha_{j}+\gamma$, so that the sum in (6.16) represents a solution of $L[y]=G(x)$ if we replace $\alpha_{j}$ by $\alpha_{j}+\gamma$. There now remains only to replace $x$ by $x+\gamma$ in the sum, and this gives (6.17).

To treat the general case, in which $F(x)$ is merely analytic, it is convenient to make a slight transformation on equation (6.3). The unique point $x^{*}$ of Lemma 5.1, which is contained in the region of convergence of all $A_{n}(x)$ expansions, may conceivably be on a critical ray (whether of first or second type). This is undesirable. Let us make the translation $x=x^{\prime}+\delta$. Then $L[y(x)]$ becomes $L_{\delta}\left[y\left(x^{\prime}\right)\right]$, where $L_{\delta}$ is determined by the function $L_{\delta}(t)=e^{\delta t} \cdot L(t)$. The zeros of $L_{\delta}(t)$ and $L(t)$ are therefore the same, so that in going from operator $L$ to $L_{\delta}$, the secondary critical rays are left unchanged. Again, the points $-\omega_{1}, \cdots,-\omega_{k}$ are replaced by $-\omega_{1}-\delta, \cdots,-\omega_{k}-\delta$, which is a translation of the points $P_{1}, \cdots, P_{k}$; hence primary rays are also unchanged. Thus, in going from $L$ to $L_{\delta}$, all critical rays are invariant. The translation on the points $P_{j}$ means that the new unique point $x_{\delta}^{*}$ is related to the old by $x_{\delta}^{*}=x^{*}-\delta$; while the minimum value $\rho^{*}$ is left unchanged: $\rho_{0}^{*}=\rho^{*}$.

It is therefore possible to choose $\delta$ so that $x_{\delta}^{*}$ is not on any critical ray. Now in transforming from $L$ to $L_{\delta}$, the form of the equation is the same. Hence: we may assume from now on that in equation (6.3), $x$ is not on any critical ray. This implies no loss of generality of equation (6.3). 
We set down the following theorem (of which we have need), due to Carmichael: $\dagger$

THEOREM 6.6. If $F(x)$ is an entire function, then equation (6.3) has an entire function solution.

7. The general case. To treat the general case where $F(x)$ is merely analytic, we follow a path suggested by $\$ 4$. For this we need a function that plays a role analogous to that played by the function $\pi \cot \pi(x-\alpha)$ of $(4.7)$. We shall be able to show that such a function exists.

Let $C$ be the unique circle, center at $x^{*}$ and radius $\rho^{*}$, assured us by Lemma 5.1. As a consequence of uniqueness there are seen to be precisely two possibilities: Either

Case I. There are at least two points $P_{j}$ on $C$, and of these at least one pair, say $P_{1}, P_{2}$, are diametrically opposite.

Or,

Case II. There are at least three points $P_{j}$ on $C$; of these no two are diametrically opposite, but at least one triad of them (say $P_{1}, P_{2}, P_{3}$ ) forms an acute-angled triangle.

Case I. Let $1,2, \cdots, k$ stand for the points $P_{1}, \cdots, P_{k}$, and let $1^{\prime}, 2^{\prime}, \cdots, k^{\prime}$ be the points respectively symmetric to $1,2, \cdots, k$ in the center $x^{*}$ of $C$. Consider the $k-1$ vectors $v_{2}, \cdots, v_{k}$ issuing from point $1^{\prime}$, along the lines $1^{\prime} 2^{\prime}, \cdots, 1^{\prime} k^{\prime}$, but in the opposite sense, and of lengths $1^{\prime} 2^{\prime}, \ldots, 1^{\prime} k^{\prime}$. To the ends of these vectors we ascribe "coordinates" of $(1,0, \cdots, 0), \cdots,(0,0, \cdots, 1)$ respectively. The point $1^{\prime}$ will have coordinates $(0,0, \cdots, 0)$. Now form a "lattice work" in the plane, with these $k-1$ vectors. We thus get a countable infinity of "points" $\left(n_{2}, n_{3}, \cdots, n_{k}\right)$, as $n_{2}, \cdots, n_{k}$ run independently through all integral values (from $-\infty$ to $+\infty)$. For example, the points $1^{\prime}, 2^{\prime}, \cdots, k^{\prime}$ have coordinates $(0,0, \cdots, 0)$, $(0,-1,0, \cdots, 0), \cdots,(0,0, \cdots, 0,-1)$. We shall refer to the diagram so obtained as Diagram 1 (for short, D.1).

Associated with it will be Diagram 2 (D.2), obtained by constructing an ordinary rectangular axis-system in the $(k-1)$-space of the coordinates $\left(n_{2}, \cdots, n_{k}\right)$. By the "point" $\left(n_{2}, \cdots, n_{k}\right)$ will be meant either the point in D. 1 reached by starting at $1^{\prime}$ and laying off the vector $n_{2} v_{2}+\cdots+n_{k} v_{k}$, or the point $\left(n_{2}, \cdots, n_{k}\right)$ in the rectangular space of D.2.

In D.2 let us place a $d o t$ at every point $\ddagger\left(n_{2}, \cdots, n_{k}\right)$ for which the corresponding point in D.1 is in or on $C$, with the exception of $(0, \cdots, 0)$ and $(-1,0,0, \cdots, 0)$. We shall say that a point so dotted is primary-dotted ( $p$ -

$\dagger$ These Transactions, loc. cit., pp. 11-13.

$\ddagger$ The numbers $n_{2}, \cdots, n_{k}$ are all integers, it is to be recalled. 
dotted, for short). As against this, we shall dot certain other points, which will be said to be s-dotted (secondary-dotted). These points are determined as follows. Let $\left(n_{2}, \cdots, n_{k}\right)$ be any point in D.2, and consider with it the $k-1$ points $\left(n_{2}, n_{3}, \cdots, n_{j-1}, n_{j}+1, n_{j+1}, \cdots, n_{k}\right), j=2,3, \cdots, k$. These $k$ points form a figure that we shall call an $L$-figure, and the points themselves will be vertices. Observe that every point $\left(n_{2}, \cdots, n_{k}\right)$ is a vertex of $k L$-figures.

Consider any $L$-figure. If no fewer than $k-1$ vertices are $p$-dotted, then we shall $s$-dot the kth vertex (if it is not already dotted). Likewise, if in any L-figure no fewer than $k-1$ vertices are dotted (each vertex may be either p-dotted or sdotted), then the remaining vertex (if not already dotted) is to be s-dotted.

This being understood, we now raise the question: Is it true that neither $(0,0, \cdots, 0)$ nor $(-1,0, \cdots, 0)$ is $s$-dotted? We shall show that the question is to be answered affirmatively. The important fact in the proof is

Property A. If $\left(n_{2}, \cdots, n_{k}\right)$ is in or on $C$, and is not at $1^{\prime}$ or $2^{\prime}$, then $\left(n_{2}^{\prime}, n_{3}, \cdots, n_{k}\right)$ is exterior to $C$ for all $\dagger n_{2}^{\prime} \neq n_{2}$.

If $k=2$, then no point $\left(n_{2}\right)$ is $p$-dotted, so that neither $(0,0)$ nor $(-1,0)$ is $s$-dotted. We may then assume that $k>2$. It is conceivable that there is a point $\left(n_{2}, \cdots, n_{k}\right)$ other than $(0, \cdots, 0)$ or $(-1,0, \cdots, 0)$, which , in D.1, coincides with $1^{\prime}$ or $2^{\prime}$. This possibility gives rise to a situation that had best be treated after we become familiar with the slightly simpler, contrary, case. We therefore suppose, at present, that the only point $\left(n_{2}, \cdots, n_{k}\right)$ coinciding with $1^{\prime}$ or $2^{\prime}$ in D.1, is, respectively, $(0, \cdots, 0),(-1,0, \cdots, 0)$.

Consider the "plane" (as we shall call it) $n_{2}=$ constant, say $n_{2}=n_{2}^{\prime}$. In this "plane" there may be a set of $p$-dots. These $p$-dots may generate some $s$-dots. Let us call an elementary figure the set of $p$-dots in $n_{2}=n_{2}^{\prime}$ plus all those $s$-dots derived from these $p$-dots (using no other $p$-dots). It is clear that all $s$-dots lying in an elementary figure lie only on $h_{2}$-lines (i.e., lines along which only the coordinate $n_{2}$ varies), which already contain a $p$-dot in the "plane" $n_{2}=n_{2}^{\prime}$. If then we draw all the $h_{2}$-lines through the $p$-dots that lie in the "plane" $n_{2}=\dot{n}_{2}^{\prime}$, these lines contain all the $s$-dots of the elementary figure determined by $n_{2}=n_{2}^{\prime}$. In particular, an $h_{2}$-line that contains no $p$-dot can contain no point of any elementary figure.

Now consider a second "plane" $n_{2}=n_{2}^{\prime \prime}$. Corresponding to its $p$-dots we get a second elementary figure, whose dots (whether $p$ - or $s$-) lie on $h_{2}$-lines that are all distinct from the $h_{2}$-lines of the previous elementary figure (Property A). It is possible that these two elementary figures (or even more such figures), when combined, give rise to $s$-dots not obtainable from any one elementary figure alone. Let us examine such a possibility. Let $a_{1}=\left(n_{2}, \cdots, n_{k}\right)$,

$\dagger$ D.1 readily shows the truth of this statement. 
$a_{2}=\left(n_{2}+1, n_{3}, \cdots, n_{k}\right), \cdots, a_{k}=\left(n_{2}, \cdots, n_{k-1}, n_{k}+1\right)$ be the vertices of an $L$-figure of which $k-1$ vertices are dotted, thus forcing the $k$ th to receive an $s$-dot. There are two possibilities:

(i) The $k$ th vertex is neither $a_{1}$ nor $a_{2}$. Then $a_{1}$ and $a_{2}$ are already dotted, and as they lie on an $h_{2}$-line, they belong to the same elementary figure. Hence $a_{2}$ (which is not $p$-dotted) could only have been $s$-dotted by the fact that the vertices $a_{1}, a_{3}, \cdots, a_{k}$ are all in this same elementary figure. That is, the $k$ th vertex that we are supposed to $s$-dot has already been dotted (either $p$ - or $s$-) in an elementary figure. We therefore get nothing new.

(ii) The $k$ th vertex is $a_{1}$ (or $a_{2}$ ). Then $a_{2}$ (or $a_{1}$ ) is already dotted, and inasmuch as $a_{2}$ (or $a_{1}$ ) already lies on an $h_{2}$-line containing a $p$-dot, the same is therefore true of $a_{1}$ (or $a_{2}$ ). Hence here again we never dot a point that is off the $h_{2}$-lines through $p$-dot points. Further, such new $s$-dots may give rise to additional $s$-dots, but never off an $h_{2}$-line through a $p$-dot.

It follows that if there exists an $h_{2}$-line containing no $p$-dots, then such a line can never receive an $s$-dot. But the line $n_{3}=n_{4}=\cdots=n_{k}=0$ is such a line. Hence $(0, \cdots, 0),(-1,0, \cdots, 0)$ will never be $s$-dotted, as was to be proved. We have supposed that no $\left(n_{2}, \cdots, n_{k}\right)$ other than $(0, \cdots, 0)$ or $(-1,0, \cdots, 0)$ lies at point $1^{\prime}$ or $2^{\prime}$ in D.1. Now suppose there is such a point $\left(n_{2}, \cdots, n_{k}\right)$, at $1^{\prime}$, say. It will then be true that for infinitely many sets of values $\left(n_{2}, \cdots, n_{k}\right)$ the corresponding point is at $1^{\prime}$. Consequently, in D.2, there will be infinitely many pairs of $p$-dots such that each pair lie on an $h_{2}$-line (and are consecutive points on such a line). Consider such a pair, say $a=\left(n_{2}-1, n_{3}, \cdots, n_{k}\right), b=\left(n_{2}, n_{3}, \cdots, n_{k}\right)$. From D.1 we see that the points $\left(n_{2}, n_{3}, \cdots, n_{k}\right)$ and $\left(n_{2}-1, n_{3}, \cdots, n_{j-1}, n_{j}+1, n_{j+1}, \cdots, n_{k}\right)$, $j=3,4, \cdots, k$, receive $p$-dots as also do the points $\left(n_{2}, n_{3}, \cdots, n_{j-1}, n_{j}-1\right.$, $\left.n_{j+1}, \cdots, n_{k}\right), j=2,3, \cdots, k$.

Corresponding to the "plane" $n_{2}=$ constant passing through $a$ there will be an elementary figure, which is independent of the fact that $b$ is $p$-dotted. (Point $b$ would receive an $s$-dot if it were not $p$-dotted.) The elementary figure for the "plane" $n_{2}=$ constant passing through $b$ may contain $s$-dots that would not have existed if $b$ were not $p$-dotted. But all such points in this elementary figure would become $s$-dots, anyway, when the two elementary figures were considered together, even if $b$ were not $p$-dotted. Hence all $s$-dots in an elementary figure still lie on $h_{2}$-lines through $p$-dots. On combining two or more elementary figures, the new $s$-dots obtained have the same property. Hence no $h_{2}$-line devoid of $p$-dots can contain $s$-dots, and again we conclude that neither $(0, \cdots, 0)$ nor $(-1,0, \cdots, 0)$ is $s$-dotted.

Case II. Here we have an acute-angled triangle 123 (i.e., $P_{1} P_{2} P_{3}$ ) inscribed in $C$. Of the angles $1 x^{*} 2,2 x^{*} 3,3 x^{*} 1$, at least two lie between $90^{\circ}$ and 
$180^{\circ}$. We suppose the indices $1,2,3$ so chosen that two such angles are $1 x^{*} 2$, $1 x^{*} 3$.

Let us again take points $1^{\prime}, \cdots, k^{\prime}$ symmetric to $1, \cdots, k$ in the center $x^{*}$, and construct a "lattice work" with the vectors $v_{2}, \cdots, v_{k}$ as was done in Case I. (Point $1^{\prime}$, for example, has again the coordinates $(0, \cdots, 0)$.) This gives us Diagram 1. Diagram 2 is constructed as before. We now make the same rules regarding $p$-dots and $s$-dots, with the single change that of all points $\left(n_{2}, \cdots, n_{k}\right)$ in or on $C$, only $(0, \cdots, 0)$ is not to be p-dotted.

We propose to show that $(0, \cdots, 0)$ is not $s$-dotted.

Suppose $k=3$. Then by Lemma 5.2 (which applies here) we know there are no points $\left(n_{2}, n_{3}\right)$ in or on $C$ other than $(0,0),(-1,0),(0,-1)$. There is therefore only one $s$-dot, namely at $(-1,-1)$. Hence $(0,0)$ is not $s$-dotted.

Now suppose $k>3$. We shall use the term $\pi$-plane to mean a plane in which only the coordinates $n_{2}, n_{3}$ vary (so that $n_{4}, \cdots, n_{k}$ are constant). Every $L$-figure has three of its vertices in a $\pi$-plane. We shall call the 2-dimensional $L$-figure, consisting of these three vertices, the base of the (original) $L$-figure. By $h_{2}\left(h_{3}\right)$ we shall mean a line along which only the coordinate $n_{2}$ (coordinate $\left.n_{3}\right)$ varies.

Consider any $\pi$-plane. It may contain some $p$-dots. Let us examine where they may be. We shall assume, for the present, that no point $\left(n_{2}, \cdots, n_{k}\right)$ is at 1 or $1^{\prime}$, except, of course, $(0, \cdots, 0)$ which is at $1^{\prime}$. Then an application of Lemma 5.3 gives us

Property B. In each $\pi$-plane one (and only one) of the following three possibilities is realized:

(a) There is no p-dot or there is just one.

(b) There are just two p-dots, and these are consecutive points on either an $h_{2}$ line or an $h_{3}$ line. (These two dots are then at two of the vertices of the base of an L-figure, one of the dots being a corner vertex.)

(c) There are two or more p-dots, all of them consecutive on a line $n_{2}+n_{3}=$ constant. Their coordinates (writing only the $n_{2}$ and $n_{3}$ coordinates) can be written: $\left(n_{2}^{\prime}, n_{3}^{\prime}\right),\left(n_{2}^{\prime}-1, n_{3}^{\prime}+1\right), \cdots,\left(n_{2}^{\prime}-r, n_{3}^{\prime}+r\right)$.

Let us now consider the possibility of $s$-dots. Suppose a given $L$-figure has $k-1 p$-dots, thus forcing an $s$-dot. From Property B, this $s$-dot must lie in the base of the $L$-figure, since no base is completely $p$-dotted. Suppose all the $s$-dots of this character have been put in, thus dotting the complete base of the corresponding $L$-figures. $\dagger$ We have thus enlarged the number of dots.

$\dagger$ In case (c) of Property B, the $s$-dots put in may themselves fill the non-corner points of a base, in which case it may be necessary to $s$-dot the new corner points. We suppose this has been done as often as is necessary. 
Call this system of $p$-dots and $s$-dots, $K$. Then it is readily seen that there exist no s-dots not in $K$.

For let $a$ be the first $s$-dot put in that does not belong to $K$. Then $a$ lies in an $L$-figure the remaining $k-1$ vertices of which are in $K$. Now $a$ cannot lie in the base of this $L$-figure, since $K$ contains all dotted points lying in a base of an $L$-figure. It must then be that in this $L$-figure (containing $a$ ) the base lies in $K$. But at least one vertex of the base is $s$-dotted, and this $s$-dot was forced by having all the other vertices already dotted (and hence in $K$ ). Therefore the assumed point $a$ does not exist.

We conclude that a point $P\left(n_{2}, \cdots, n_{k}\right)$ cannot be $s$-dotted if, when we consider only the 2-dimensional $L$-figures lying in the $\pi$-plane through $P$, then $P$ is not $s$-dotted. Now the $\pi$-plane for which $n_{4}=\cdots=n_{k}=0$ contains the point $(0, \cdots, 0)$, and in it only the points $(0,-1,0, \cdots, 0)$, $(-1,0, \cdots, 0)$ are $p$-dotted. Hence in this $\pi$-plane the only point that could possibly be $s$-dotted is $(-1,-1,0, \cdots, 0)$. In particular, then, $(0, \cdots, 0)$ is never $s$-dotted.

We may now remove the restriction that no point $\left(n_{2}, \cdots, n_{k}\right)$ can lie at 1 or $1^{\prime}$ other than $(0, \cdots, 0)$ at $1^{\prime}$. This now permits the existence of infinitely many $\pi$-planes that contain three $p$-dots. $\dagger$ This increase in $p$-dots may permit more $s$-dots than was the case before, but as was true in Case I, the slight increase in complexity will not alter the fact that $(0, \cdots, 0)$ remains free of an $s$-dot.

Summing up, we have

Lemma 7.1. If p-dots are placed in Diagram 2 at all points $\left(n_{2}, \cdots, n_{k}\right)$ that are in or on $C$, save $(0, \cdots, 0),(-1,0, \cdots, 0)$ in Case $I$ and save $(0, \cdots, 0)$ in Case II, and if s-dots are placed in accordance with the L-figure rule, then $(0, \cdots, 0),(-1,0, \cdots, 0)$ remain free of a dot in Case I and $(0, \cdots, 0)$ remains free of a dot in Case II.

Consider the homogeneous equation

$$
L[Y(x ; \alpha)]=0 .
$$

Assume a formal solution

(7.2) $\quad Y(x ; \alpha) \sim \sum_{n_{2}, n_{3}, \cdots, n_{k}=-\infty}^{+\infty} \overline{\left[x-\left(\alpha+\overline{\omega_{1}}\right)-n_{2}\left(\omega_{1}-\omega_{2}\right)-b_{n_{2}, n_{3}, \cdots, n_{k}}-n_{3}\left(\omega_{1}-\omega_{3}\right)-\cdots-n_{k}\left(\omega_{1}-\omega_{k}\right)\right]}$,

† If $\left(n_{2}, \cdots, n_{k}\right)$ is at 1 , the three $p$-dots make up the base of an $L$-figure; but if $\left(n_{2}, \cdots, n_{k}\right)$ is at $1^{\prime}$, two of the $p$-dots are non-corner points of a base, while the third is the vertex opposite to the zorner point in the unit square that contains the base. 
where $-\omega_{1}$ is the point $P_{1}$ of Case I or Case II. On substituting into (7.1) we obtain

$$
0=\sum_{n_{2}, \cdots n_{k}-\infty}^{+\infty} \frac{a_{1} b_{n_{2}}, \cdots, n_{k}+a_{2} b_{n_{2}+1, n_{3}, \cdots, n_{k}}+a_{3} b_{n_{2}, n_{3}+1, \cdots, n_{k}}+\cdots+a_{k} b_{n_{2}}, \cdots, n_{k-1}, n_{k}+1}{\left[x-\alpha-\sum_{j=2}^{k} n_{j}\left(\omega_{1}-\omega_{j}\right)\right]},
$$

and this is formally satisfied if the $b$ 's are chosen so that

$$
\begin{aligned}
a_{1} b_{n_{2}}, \cdots, n_{k} & +\sum_{j=2}^{k} a_{j} b_{n_{2}, \cdots, n_{j-1}, n_{j}+1, n_{j+1}, \cdots, n_{k}}=0 \\
\left(n_{2}, n_{3}, \cdots, n_{k}\right. & =0, \pm 1, \pm 2, \cdots) .
\end{aligned}
$$

Choose $\alpha=x^{*}$ and let

$$
\tau_{n_{2} \cdots n_{k}}=x^{*}+\omega_{1}+\sum_{j=2}^{k} n_{j}\left(\omega_{1}-\omega_{j}\right)
$$

The poles of $Y\left(x ; x^{*}\right)$ (in a purely formal way) are among the points $\tau$. Now $x^{*}+\omega_{1}$ is at a distance $\rho^{*}$ from the origin, and the line joining it to the origin is parallel to $11^{\prime}$ in D.1. Hence those $\tau$ 's which are at a distance not exceeding $\rho^{*}$ from the origin correspond to those vectors

$$
\lambda_{n_{2} \cdots n_{k}}=\sum_{j=2}^{k} n_{j}\left(\omega_{1}-\omega_{j}\right)
$$

which, when laid off with initial point at $1^{\prime}$, have their terminal point in or on $C$. Now when so laid off, the $\lambda$ 's give us precisely the points $\left(n_{2}, \cdots, n_{k}\right)$ of the lattice work of Lemma 7.1. If we wish not to have poles $\tau$ at a distance not exceeding $\rho^{*}$ from the origin, then we must choose $b_{n_{2} \ldots n_{k}}=0$ for every $\left(n_{2}, \cdots, n_{k}\right)$ in or on $C$ (in D.1). We shall, however, except the values $(0, \cdots, 0),(-1,0, \cdots, 0)$ in Case I, and the value $(0, \cdots, 0)$ in Case II (i.e., the corresponding $b$ 's are (or $b$ is) not to be chosen zero). Otherwise put, all $b$ 's corresponding to $p$-dots are to be chosen zero. Now on so doing (7.3) informs us that whenever $k-1 b$ 's, corresponding to $k-1$ vertices of an $L$-figure, are zero, we must choose the $k$ th $b$ also to be zero. That is, $b$ 's corresponding to $s$-dots are also to be chosen zero. In the light of Lemma 7.1 we have

Lemma 7.2. Constants $b_{n_{2} \ldots n_{k}}$ exist such that there is a formal series (7.2), with $\alpha=x^{*}$, satisfying (7.1), and such that

(i) $b_{0, \ldots, 0} \neq 0, b_{-1,0, \ldots, 0} \neq 0$ in Case I, and $b_{0, \ldots, 0} \neq 0$ in Case II;

(ii) no formal pole of $Y\left(x ; x^{*}\right)$ is at a distance not exceeding $\rho^{*}$ from the origin, save $\tau_{0, \ldots, 0}, \tau_{-1,0, \ldots, 0}$ in Case I and save $\tau_{0, \ldots, 0}$ in Case II. 
We shall hereafter assume that the $b_{n_{2} \ldots n_{k}}$ 's have been chosen to satisfy Lemma 7.2. Also, from the homogeneous character of equations (7.3), we may suppose that $b_{0, \ldots, 0}=-1 / \alpha_{1}$. We dare not expect that series (7.2) is convergent. But by the classic theorem of Mittag-Leffler, there exist polynomials $P_{n_{2} \ldots n_{k}}(x)$ such that the series

$$
Z\left(x ; x^{*}\right)=\sum_{n_{2}, \cdots, n_{k}=-\infty}^{+\infty}\left(\frac{b_{n_{2} \cdots n_{k}}}{\left[x-\left(x^{*}+\omega_{1}\right)-\sum_{j=2}^{k} n_{j}\left(\omega_{1}-\omega_{j}\right)\right]}+P_{n_{2} \cdots n_{k}}(x)\right)
$$

converges uniformly and absolutely in every bounded region, the zeros of the denominators deleted. $\dagger$ The operator $L$ may be applied term-wise to (7.6), valid for every $x$ not a pole; and the only possible singularities of $L[Z]$ are at the points $\tau_{n_{2}}, \ldots, n_{k}$. Now on suitably grouping terms in $L[Z]$, as is permissible because of absolute convergence, we get

$$
\begin{aligned}
& L[Z]=\sum_{n_{2}, \cdots, n_{k}=-\infty}^{+\infty}\left(\frac{A_{n_{2} \cdots n_{k}}}{\left[x-x^{*}-\sum_{j=2}^{k} n_{j}\left(\omega_{1}-\omega_{j}\right)\right]}+a_{1} P_{n_{2} \cdots n_{k}}\left(x+\omega_{1}\right)\right.
\end{aligned}
$$

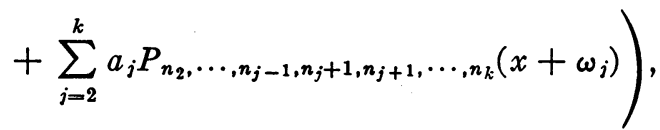

where $A_{n_{2} \ldots n_{k}}$ is the left-hand member of (7.3), and is accordingly zero. Hence $L[Z]$ does not have any poles. $\ddagger$ That is, $L[Z]$ is an entire function. Now by Theorem 6.6 there is an entire function $g(x)$ satisfying

$$
L[g(x)]=L\left[Z\left(x ; x^{*}\right)\right],
$$

so that the function $W\left(x ; x^{*}\right)=Z\left(x ; x^{*}\right)-g(x)$ satisfies

$$
L\left[W\left(x ; x^{*}\right)\right]=0 .
$$

We therefore have

THEOREM 7.1.§ The homogeneous equation (7.7) has a meromorphic solution $W\left(x ; x^{*}\right)$ which has a pole at $x=x^{*}+\omega_{1}$, and which has no other pole whose distance from the origin does not exceed $\rho^{*}$, except in Case $\mathrm{I}$ when there may be a pole at $x=x^{*}+\omega_{2}$.

$\dagger Z\left(x ; x^{*}\right)$ has then no pole at a distance not exceeding $\rho^{*}$ from the origin save for $\tau_{0, \cdots, 0}$, $\tau_{-1,0, \ldots, 0}$ in Case I and save for $\tau_{0, \ldots, 0}$ in Case II.

$\ddagger$ For if in the series for $Z$ (and therefore for $L[Z]$ ), any term is omitted, the resulting series converges even at the pole corresponding to the omitted term.

$\S$ In $\S 4$ the function $\Omega(x ; \alpha)$ was already at hand. It has been the purpose of Lemma 7.1 to enable us to assert the existence of a similar function in the present case. 
In Theorem 6.4 we considered $\alpha$ in a sector $\Sigma_{M}$ bounded by primary rays. From the nature of primary rays it is readily seen that to every $j$ for which the point $P_{j}$ is on $C$ there certainly corresponds a $\Sigma_{j}$-sector. Since point $P_{1}$ is on $C$, there is therefore a $\Sigma_{1}$-sector; and from a previous observation, we may suppose with no loss of generality that $x^{*}$, and in fact the whole of circle $C$, is in $\Sigma_{1}$.

Consider Case I. Let $\rho^{\prime}$ be a number only slightly $\dagger$ larger than $\rho^{*}$, and with $P_{1}$ and $P_{2}$ (i.e., $-\omega_{1}$ and $-\omega_{2}$ ) as centers, draw arcs of radius $\rho^{\prime}$. They will form a lens-contour, the two arcs of which we shall call $A_{1}, A_{2}\left(A_{j}\right.$ being part of the circle with center at $\left.P_{j}\right)$. The function $y(x ; \alpha)$ of Theorem 6.4 (with $M=1$ in the present case) has as its pole nearest the origin the point $x=\alpha+\omega_{1}$. For $\alpha=x^{*}$, this pole is at a distance $\rho^{*}$ from $x=0$, and no other pole is within this distance of $x=0$.

Now let $\alpha$ trace the lens $A$ (consisting of the $\operatorname{arcs} A_{1}, A_{2}$ ). Then $\alpha+\omega_{1}$ describes a congruent lens $A^{\prime}$, obtained from $A$ by a translation so that the new center is at $x^{*}+\omega_{1}$ instead of at $x^{*}$. Let the corresponding arcs of the new lens be $A_{1}^{\prime}, A_{2}^{\prime}$. One of these, namely $A_{1}^{\prime}$, is an arc of a circle of center $x=0$ and radius $\rho^{\prime}$, and consequently is wholly exterior to the circle of radius $\rho^{*}$. That is, for all $\alpha$ on $A_{1}, y(x ; \alpha)$ has no singularity at a distance from $x=0$ less than or equal to $\rho^{*}$. If we write

$$
y(x ; \alpha)=\sum_{0}^{\infty} c_{n}(\alpha) x^{n},
$$

this series converges uniformly in $\alpha$ and $x$ for all $\alpha$ on $A_{1}$ and all $x$ in $|x| \leqq \rho^{*}+\epsilon(\epsilon>0$ sufficiently small $)$.

Now let $\alpha$ be on $A_{2}$. If in $Y\left(x ; x^{*}\right)$ we replace $x^{*}$ by $\alpha$, the result is to give us the function $W(x ; \alpha)$ obtained from $W\left(x ; x^{*}\right)$ by replacing $x^{*}$ by $\alpha$ in $Z\left(x ; x^{*}\right)$. Inasmuch as $x^{*}$ and $x$ enter $W\left(x ; x^{*}\right)$ only in their difference $x-x^{*}$, we have

$$
W(x ; \alpha)=W\left(x+x^{*}-\alpha ; x^{*}\right) .
$$

Consider the function

$$
y_{1}(x ; \alpha)=y(x ; \alpha)+W(x ; \alpha) .
$$

For $\rho^{\prime}$ sufficiently near to $\rho^{*}$, and for $\alpha$ on $A_{2}$, the only possible poles of $y_{1}(x ; \alpha)$ whose distance from $x=0$ does not exceed $\rho^{*}$ are at $x=\alpha+\omega_{1}$, $x=\alpha+\omega_{2}$, since this is true separately of $y(x ; \alpha)$ and $W(x ; \alpha)$.

Consider the point $x=\alpha+\omega_{1}$. At this point $y(x ; \alpha)$ has a simple pole with

$\dagger$ Just how close to $\rho^{*}$ it must be taken will soon appear. 
multiplier $1 / a_{1}$. But also $W(x ; \alpha)$ has a simple pole there, with a multiplier $b_{0}, \ldots, 0$ which we chose equal to $-1 / a_{1}$. Hence $y_{1}(x ; \alpha)$ remains analytic at $x=\alpha+\omega_{1}$.

Now let us examine $x=\alpha+\omega_{2}$. The point $x^{*}+\omega_{2}$ is at a distance $\rho^{*}$ from $x=0$, and is diametrically opposite to $x^{*}+\omega_{1}$, from $x=0$. Hence as $\alpha$ traces $\operatorname{arc} A_{2}$, the point $\alpha+\omega_{2}$ traces an arc of a circle of radius $\rho^{\prime}$, center at $x=0$. Hence even if $\alpha+\omega_{2}$ is a pole of $y_{1}(x ; \alpha)$, it lies at a distance greater than $\rho^{*}$ from $x=0$. Then,

$$
y_{1}(x ; \alpha)=\sum_{0}^{\infty} d_{n}(\alpha) x^{n},
$$

uniformly convergent in $\alpha$ and $x$ for all $\alpha$ on $A_{2}$ and for all $x$ in $|x| \leqq \rho^{*}+\epsilon$, $\epsilon>0$ sufficiently small.

We have

$$
\lim \sup \left|c_{n}(\alpha)\right|^{1 / n} \leqq \frac{1}{\rho^{*}+\epsilon}, \quad \lim \sup \left|d_{n}(\alpha)\right|^{1 / n} \leqq \frac{1}{\rho^{*}+\epsilon},
$$

so that we can operate on (7.8) and (7.12) with $L$, getting $\dagger$

$$
L[y(x ; \alpha)]=\sum_{0}^{\infty} c_{n}(\alpha) A_{n}(x), \quad L\left[y_{1}(x ; \alpha)\right]=\sum_{0}^{\infty} d_{n}(\alpha) A_{n}(x),
$$

the series being uniformly convergent for all $\alpha, x$ such that $\alpha$ is on $A_{1}, A_{2}$ respectively, and $x$ is in a closed region containing $x^{*}$ and of diameter sufficiently small. But $L[W]=0$, so that $L[y]=L\left[y_{1}\right]=1 /(x-\alpha)$. Hence

$$
\frac{1}{x-\alpha}=\sum_{n=0}^{\infty} c_{n}(\alpha) A_{n}(x), \quad \frac{1}{x-\alpha}=\sum_{n=0}^{\infty} d_{n}(\alpha) A_{n}(x),
$$

uniformly convergent for all $\alpha$ and $x$ such that $\alpha$ is on $A_{1}, A_{2}$ respectively, and $x$ is in a small enough neighborhood of $x=x^{*}$.

Now we take up Case II. $\rho^{\prime}$ being a slightly larger number than $\rho^{*}$, we strike three $\operatorname{arcs} A_{1}, A_{2}, A_{3}$ of radius $\rho^{\prime}$ and centers $-\omega_{1},-\omega_{2},-\omega_{3}$, forming a curvilinear triangle. When $\alpha$ traces $A_{1}$, the point $\alpha+\omega_{1}$ traces a congruent arc with center at $x=0$, so that the pole $\alpha+\omega_{1}$ (which is the nearest pole to the origin) of $y(x ; \alpha)$ is at a distance from $x=0$ exceeding $\rho^{*}$. Hence (7.8) continues to hold, $\alpha$ remaining on $A_{1}$. For $\alpha$ on either $A_{2}$ or $A_{3}$, the function $y_{1}(x ; \alpha)$ of $(7.10)$ has no singularity at $x=\alpha+\omega_{1}$ (as in Case I). But (cf. Theorem 7.1) $y_{1}(x ; \alpha)$ can have no other singularity within a distance $\rho^{*}$ of $x=0$. 
Hence there are no singularities within this distance, and (7.11) holds uniformly for $\alpha$ on $A_{2}+A_{3}$. From this follows the continued validity of (7.13) and (7.14), for $\alpha$ on $A_{1}$ and on $A_{2}, A_{3}$ respectively. That is, we have

THEOREM 7.2. The function $1 /(x-\alpha)$ has the $A_{n}$-expansions of (7.14), uniformly convergent, respectively, for all $\alpha$ on $A_{1}$ and all $\alpha$ on $A_{2}$ in Case I (and all $\alpha$ on $A_{2}$ and $A_{3}$ in Case II), and for all $x$ in a sufficiently small neighborhood of $x=x^{*}$.

Now let $F(x)$ be analytic about $x=x^{*}$. Then there exists (according to Case I or Case II) a lens or circular triangle around $x^{*}$ lying with its boundary wholly in the region of analyticity of $F(x)$. On applying the Cauchy formula to series (7.14), term-by-term integration being of course allowable, we obtain

$$
F(x)=\sum_{0}^{\infty} f_{n} A_{n}(x)
$$

where

$$
f_{n}=\left\{-\frac{1}{2 \pi i} \int_{A_{1}} c_{n}(\alpha) F(\alpha) d \alpha-\frac{1}{2 \pi i} \int_{A_{2} \text { or } A_{2}+A_{3}} d_{n}(\alpha) F(\alpha) d \alpha\right\},
$$

the series being uniformly convergent for $x$ sufficiently close to $x^{*}$. Hence

THEOREM 7.3. If $F(x)$ is analytic about $x=x^{*}$, it has a convergent $A_{n}$-expansion, given by (7.15).

Combining this with Theorem 6.1:

THEOREM 7.4. A necessary and sufficient condition that a function $F(x)$ have $a$ (convergent) $A_{n}$-expansion is that it be analytic at $x=x^{*}$.

By Theorem 6.2, lim sup $\left|f_{n}\right|^{1 / n}<1 / \rho^{*}$, so that the series

$$
y(x)=\sum_{0}^{\infty} f_{n} x^{n}
$$

converges in $|x|<\rho^{*}+\epsilon$, for some $\epsilon>0$. On applying $L$ to (7.17) we get

$$
L[y(x)]=\sum f_{n} L\left[x^{n}\right]=\sum f_{n} A_{n}(x)=F(x),
$$

so that

THEOREM 7.5. If $F(x)$ is analytic about $x=x^{*}$, then the function $y(x)$ of (7.17) is analytic in a circle about $x=0$ of radius greater than $\rho^{*}$, and for all $x$ in this circle $y(x)$ satisfies the equation

$$
L[y(x)]=F(x) .
$$


The point $x^{*}$ is of course significant for $A_{n}$-expansions, but not for equation (6.3). For let $F(x)$ be analytic about $x=c$, and define $G(x)=F\left(x+c-x^{*}\right)$. $G(x)$ is analytic about $x=x^{*}$ and therefore there exists a function $z(x)$ such that $L[z(x)]=G(x)$. Consequently, the function $y(x)=z\left(x-c+x^{*}\right)$ satisfies $L[y(x)]=F(x)$, and we have the final

THEOREM 7.6. If $F(x)$ is analytic about $x=c$, there exists a function $y(x)$, analytic about $x=c-x^{*}$ in a circle of radius exceeding $\rho^{*}$, such that for all $x$ in this circle $y(x)$ satisfies equation (6.3).

Pennsyluania State College, State College, Pa. 